\title{
Spin relaxation in a complex environment
}

\author{
Massimiliano Esposito and Pierre Gaspard \\ Center for Nonlinear Phenomena and Complex Systems, Université Libre de Bruxelles, Code Postal 231, \\ Campus Plaine, B-1050 Brussels, Belgium
}

(Received 6 August 2003; published 24 December 2003)

\begin{abstract}
We report the study of a model of a two-level system interacting in a nondiagonal way with a complex environment described by Gaussian orthogonal random matrices (GORM). The effect of the interaction on the total spectrum and its consequences on the dynamics of the two-level system is analyzed. We show the existence of a critical value of the interaction, depending on the mean level spacing of the environment, above which the dynamics is self-averaging and closely obey a master equation for the time evolution of the observables of the two-level system. Analytic results are also obtained in the strong coupling regimes. We finally study the equilibrium values of the two-level system population and show under which condition it thermalizes to the environment temperature.
\end{abstract}

DOI: 10.1103/PhysRevE.68.066113

PACS number(s): 05.50.+q, 03.65.Yz

\section{INTRODUCTION}

The nonequilibrium statistical mechanics of small quantum systems has become a topic of fundamental importance for nanosciences. It is indeed very important to understand what the minimum sizes and conditions are under which a quantum system can display a relaxation to an equilibrium state. Isolated and finite quantum systems have a discrete energy spectrum, which has for consequence that all the observables present almost periodic recurrences on long time scales. Nevertheless, their early time evolution may still present relaxation types of behavior that is important to study. Although tools have been developed in nonequilibrium statistical mechanics to describe such relaxations to a state of equilibrium by master and kinetic equations, the conditions of validity of these equations remain little known.

It is the purpose of this paper to contribute to the clarification of these questions of validity of the kinetic description by studying a simple model of a two-level system or spin coupled to a complex environment described by random matrices. Indeed, work done during the last decade has shown that the Hamiltonian of typical quantum systems presents properties of random matrices on their small energy scales. Here, we consider the Hamiltonian of the environment as well as the operator of coupling between the spin and the environment to be given by random matrices taken in a statistical ensemble of Gaussian orthogonal random matrices. This defines a model in which many results can be obtained analytically.

Similar models using Gaussian orthogonal random matrices $[1,2]$ or using banded random matrices [3-7] have been studied. In [5], it has been shown that random matrices used as environment coupling operators have a universal feature.

The model we here consider differs from the spin-boson model by the density of states of the environment. Instead of monotonously increasing with energy as in the spinboson model, the density of states of the environment obeys Wigner's semicircular law in our model and is thus limited to an interval of energy with a maximum density in between. We notice that such densities of states appear in systems where a spin is coupled to other (possibly dissimilar) spins as, for instance, in NMR, in which case the density of states of the other spins forming the environment also present a maximum instead of a monotonous increase with energy. Our model may therefore constitute a simplification of such kinds of interacting spin systems. Our main purpose is to understand the conditions under which a kinetic description can be used in order to understand the relaxation of the spin under the effect of the coupling with the rest of the system, which we refer to as a complex environment.

The plan of the paper is the following. The model is presented in Sec. II. The properties of the spectrum are described in Sec. III. The relaxation in the time evolution of the spin is studied in Sec. IV. The very long time behavior and the approach to the equilibrium is discussed in Sec. V. Conclusions are drawn in Sec. VI.

\section{THE MODEL}

We are interested in the study of a total system composed of a simple system (with a few discrete levels) interacting with a complex environment (with many levels). We consider a two-level system as a prototype for the simple system.

For this kind of total system, the time-dependent Schrödinger equation is of the following type:

$$
i \hbar \frac{d|\Psi(\tilde{t})\rangle}{d \tilde{t}}=\hat{\tilde{H}}_{\text {tot }}|\Psi(\tilde{t})\rangle=\left(\frac{\widetilde{\Delta}}{2} \hat{\sigma}_{z}+\hat{\tilde{H}}_{B}+\tilde{\lambda} \hat{\sigma}_{x} \hat{\tilde{B}}\right)|\Psi(\tilde{t})\rangle,
$$

where $\hat{\sigma}_{x}, \hat{\sigma}_{y}$, and $\hat{\sigma}_{z}$ are the $2 \times 2$ Pauli matrices, $(\widetilde{\Delta} / 2) \hat{\sigma}_{z}$ is the Hamiltonian of the two-level system, $\widetilde{\Delta}$ is the energy spacing between the two levels of the system, $\hat{\tilde{H}}_{B}$ is the Hamiltonian of the environment, $\hat{\sigma}_{x}$ is the coupling operator of the system, $\hat{\widetilde{B}}$ is the coupling operator of the environment, and $\tilde{\lambda}$ is the coupling parameter between the system and the environment.

The well-known spin-boson model [8-10] is a particular case of the total system where $\hat{\tilde{H}}_{B}$ corresponds to an infinite 
harmonic oscillator lattice and $\hat{\widetilde{B}}$ is linear in the degree of freedom of the environment. Here, we want to define a new model, the spin-GORM model, also described by the Hamiltonian (1) and for which $\widehat{H}_{B}$ and $\hat{B}$ are Gaussian orthogonal random matrices (GORM) (see Appendix A for some basic property on GORM).

Let us discuss now the spin-GORM model in more detail. As we said, we want to model a two-level system that interacts with an environment that has a complex dynamics. Here, complex is used in a generic way. The complexity can come, for example, from the fact that the corresponding classical system is chaotic like in a quantum billiard or for the hydrogen atom in a strong magnetic field $[11,12]$. It can also come from large coupling in an interacting many-body system as in nuclear physics [11] or in interacting fermion systems such as quantum computers [11,13]. Wigner in 1960 [14-16] was the first to develop random-matrix theory for the purpose of modeling spectral fluctuations of complex quantum systems containing many states interacting with each other. This tool has now become very common in many fields from nuclear physics to quantum chaos. This is the reason why we consider random matrices to characterize the complexity of the environment operators.

The environment operators of the spin-GORM model, $\hat{\widetilde{H}}_{B}$ and $\widehat{B}$, are defined by

$$
\begin{aligned}
& \hat{H}_{B}=\sigma_{N D}^{\hat{\hat{H}}_{B}} \hat{X} \\
& \hat{B}=\sigma_{N D}^{\hat{\tilde{B}}} \hat{X}^{\prime},
\end{aligned}
$$

where $\hat{X}$ and $\hat{X}^{\prime}$ are two different $(N / 2) \times(N / 2)$ Gaussian orthogonal random matrices with mean zero. Their nondiagonal (diagonal) elements have standard deviation $\sigma_{N D}^{\hat{\hat{X}}}=1$ $\left(\sigma_{D}^{\hat{\hat{X}}}=\sqrt{2}\right) . \hat{X}$ and $\hat{X}^{\prime}$ are two different realizations of the same random matrix ensemble and have therefore the same statistical properties. $\sigma_{N D}^{\hat{\hat{H}}_{B}}$ and $\sigma_{N D}^{\hat{\hat{B}}}$ are the standard deviations of the nondiagonal elements of $\hat{\widetilde{H}}_{B}$ and $\hat{\widetilde{B}}$, respectively. For these random matrices, the width of their averaged smoothed density of state is given by

$$
\begin{aligned}
\mathcal{D} \widetilde{H}_{B} & =\sigma_{N D}^{\hat{\tilde{H}}_{B}} \sqrt{8 N}, \\
\mathcal{D} \widetilde{B} & =\sigma_{N D}^{\hat{\tilde{B}}} \sqrt{8 N}
\end{aligned}
$$

(see Appendix A).

It is interesting to define the model in such a way that, when $N$ is increased, the averaged smoothed density of state of the environment increases without changing its width $\mathcal{D} \widetilde{H}_{B}$. The width can be fixed to unity. This is equivalent to fixing the characteristic time scale of the environment. For doing this, it is necessary to rescale the parameters as follows:

$$
\alpha=\sigma_{N D}^{\hat{\hat{H}}_{B}} \sqrt{8 N}
$$

$$
\begin{gathered}
t=\alpha \widetilde{t}, \\
\Delta=\frac{\widetilde{\Delta}}{\sigma_{N D}^{\hat{\tilde{H}}_{B} \sqrt{8 N}}}, \\
\lambda=\tilde{\lambda} \frac{\sigma_{N D}^{\hat{\tilde{B}}}}{\sigma_{N D}^{\hat{\tilde{H}}_{B}}} \\
N=N .
\end{gathered}
$$

The time-dependent Schrödinger equation of the spinGORM model becomes

$$
i \hbar \frac{d|\Psi(t)\rangle}{d t}=\hat{H}_{\mathrm{tot}}|\Psi(t)\rangle,
$$

with the rescaled total Hamiltonian

$$
\hat{H}_{\mathrm{tot}}=\hat{H}_{S}+\hat{H}_{B}+\lambda \hat{\sigma}_{x} \hat{B}=\frac{\Delta}{2} \hat{\sigma}_{z}+\frac{1}{\sqrt{8 N}} \hat{X}+\lambda \hat{\sigma}_{x} \frac{1}{\sqrt{8 N}} \hat{X}^{\prime} .
$$

As announced, we have now $\mathcal{D} H_{B}=\mathcal{D} B=1$.

In the following, without loss of generality, $\alpha$ will always be taken equal to unity. Notice that, to model an environment with a quasicontinuous spectrum, the random matrix must be very large $(N \rightarrow \infty)$.

In order to get ensemble averaged results, one has to perform averages over the different results obtained for each realization of Eq. (5). When we use finite ensemble averages, the number of members of the ensemble average will be denoted by $\chi$.

We see that the Hamiltonian (5) is characterized by three different parameters: $\Delta, \lambda$, and $N$. We define three different parameter domains in the reduced parameter space corresponding to a fixed $N$ in order to facilitate the following discussion. These three regimes are represented in Fig. 1: domain A with $1>\lambda, \Delta$; domain B with $\Delta>1, \lambda$; domain $\mathrm{C}$ with $\lambda>1, \Delta$.

\section{THE SPECTRUM}

In this section we study the spectrum of the complete system for the different values of the parameters. This study is important in order to understand the different dynamical behaviors that we encounter in the model.

Let us begin defining the notations in the simple case where there is no coupling between the two parts of the total system $(\lambda \rightarrow 0)$. The isolated system has two levels separated by the energy $\Delta$ :

$$
\hat{H}_{S}|s\rangle=s \frac{\Delta}{2}|s\rangle,
$$

where $s= \pm 1$. The environment has the standard spectrum of a GORM 


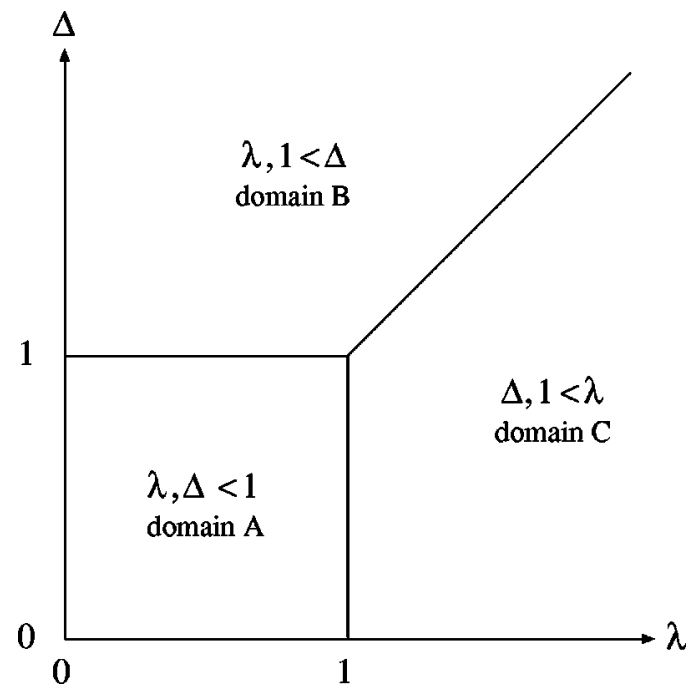

FIG. 1. Representation of the three different domains in the space of the reduced parameters $\lambda$ and $\Delta$ of the model for a fixed number $N$ of states.

$$
\hat{H}_{B}|b\rangle=E_{b}^{B}|b\rangle,
$$

where $b=1,2, \ldots, N / 2$. The Hamiltonian of the total system without interaction between the system and the environment is thus

$$
\hat{H}_{0}=\hat{H}_{S}+\hat{H}_{B},
$$

and the spectrum is therefore given by

$$
\hat{H}_{0}|n\rangle=E_{n}^{0}|n\rangle,
$$

with $n=1,2, \ldots, N$ and

$$
E_{n}^{0}=s \frac{\Delta}{2}+E_{b}^{B} .
$$

The eigenvectors are tensorial products of both the system and environment eigenvectors:

$$
|n\rangle=|s\rangle \otimes|b\rangle .
$$

Let us now define the notations in the opposite simple situation where the coupling term is so large that the Hamiltonian of the system and of the environment can both be neglected $(\lambda \rightarrow \infty)$. Using the unitary matrix $\hat{U}$ acting only on the system degrees of freedom

$$
\hat{U}=\left[\begin{array}{cc}
\frac{1}{\sqrt{2}} & \frac{1}{\sqrt{2}} \\
\frac{1}{\sqrt{2}} & -\frac{1}{\sqrt{2}}
\end{array}\right]
$$

the total Hamiltonian becomes

$$
\widetilde{\hat{H}}_{0}=\lambda \hat{\sigma}_{z} \hat{B} .
$$

$E_{\kappa \eta}$ and $|\kappa \eta\rangle=|\kappa\rangle \otimes|\eta\rangle$ are, respectively, the eigenvalues and eigenvectors of the Hamiltonian

$$
\widetilde{H}_{0}|\kappa \eta\rangle=\lambda E_{\kappa \eta}|\kappa \eta\rangle=\lambda \kappa E_{\eta}|\kappa \eta\rangle,
$$

where $\eta=1, \ldots, N / 2$ and $\kappa= \pm 1$. After having defined the notation in the two extreme cases $\lambda \rightarrow 0$ and $\lambda \rightarrow \infty$, we will start the study of the spectrum with interaction $\lambda \neq 0$.

The total spectrum is given by the eigenvalues $\left\{E_{\alpha}\right\}$ which are solutions of the eigenvalue problem

$$
\hat{H}_{\text {tot }}|\alpha\rangle=E_{\alpha}|\alpha\rangle,
$$

where $\alpha=1,2, \ldots, N$. It is very difficult to obtain analytical results for this problem. We will therefore study the total spectra using a method of numerical diagonalization of the total Hamiltonian.

\section{A. Smoothed density of states}

In order to have a quantitative understanding of the global aspect of the spectrum (on large energy scales), we will study the total perturbed averaged smoothed density of states.

The environment-averaged smoothed density of states obeys the semicircular Wigner law [see Eq. (A4) in Appen$\operatorname{dix}$ A]

$$
n^{w}(\epsilon)= \begin{cases}\frac{4 N}{\pi} \sqrt{\left(\frac{1}{2}\right)^{2}-\epsilon^{2}} & \text { if }|\epsilon|<\frac{1}{2}, \\ 0 & \text { if }|\epsilon| \geqslant \frac{1}{2},\end{cases}
$$

where $\epsilon$ is the continuous variable corresponding to the environment energy $E_{b}^{B}$.

Therefore, when $\lambda=0$, the total averaged smoothed density of states is the sum of the two environment semicircular densities of states which correspond to both states of the two-level system [see Eq. (9)]:

$$
n(\varepsilon)=n^{w}\left(\varepsilon-\frac{\Delta}{2}\right)+n^{w}\left(\varepsilon+\frac{\Delta}{2}\right)=\left\{\begin{array}{l}
\frac{4 N}{\pi} \sqrt{\left(\frac{1}{2}\right)^{2}-\left(\varepsilon-\frac{\Delta}{2}\right)^{2}}+\frac{4 N}{\pi} \sqrt{\left(\frac{1}{2}\right)^{2}-\left(\varepsilon+\frac{\Delta}{2}\right)^{2}} \\
\text { if }\left(\frac{1}{2}-\frac{\Delta}{2}\right)<|\varepsilon|<\left(\frac{1}{2}+\frac{\Delta}{2}\right), \\
0 \quad \text { elsewhere, }
\end{array}\right.
$$




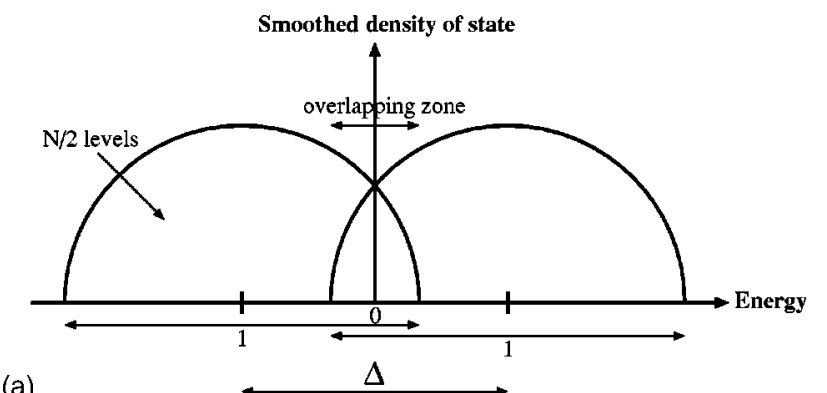

(a)

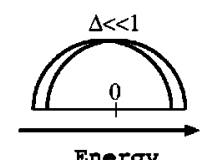

(b)

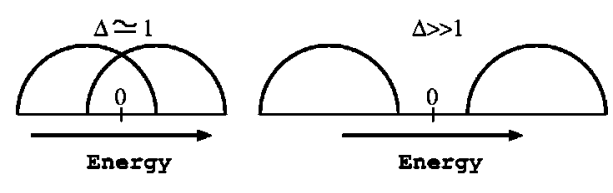

FIG. 2. Smoothed densities of states $n^{w}[\varepsilon-(\Delta / 2)]$ and $n^{w}[\varepsilon$ $+(\Delta / 2)]$ for different values of $\Delta$. The total smoothed averaged density of states of the nonperturbed spectrum is obtained by the sum of them [see Eq. (16)].

where $\varepsilon$ is the continuous variable corresponding to the total energy $E_{n}^{0}$. The semicircular densities $n^{w}[\varepsilon-(\Delta / 2)]$ and $n^{w}[\varepsilon+(\Delta / 2)]$ are schematically depicted in Fig. 2 for different values of $\Delta$. The numerical density of states of the total system corresponding to $\lambda=0$ is depicted in Fig. 3 .

When $\lambda \rightarrow \infty$ (meaning that the coupling term becomes dominant in the Hamiltonian), the averaged smoothed density of states of the total system [see Eq. (13)] is given by

$$
\begin{aligned}
n(\varepsilon) & =n^{w}\left(\frac{\varepsilon}{\lambda}\right)+n^{w}\left(-\frac{\varepsilon}{\lambda}\right) \\
& =\left\{\begin{array}{l}
\frac{8 N}{\lambda \pi} \sqrt{\left(\frac{\lambda}{2}\right)^{2}-(\varepsilon)^{2}} \text { if }|\varepsilon|<\frac{\lambda}{2}, \\
0 \text { if }|\varepsilon| \geqslant \frac{\lambda}{2},
\end{array}\right.
\end{aligned}
$$

where $\varepsilon$ is the continuous variable corresponding to the total energy $E_{\kappa \eta}$. This result can be observed in Fig. 3(a) for $\lambda=10$ (because $\Delta, 1 \ll \lambda$ ).

When $\lambda \neq 0$, the total averaged smoothed density of states is also plotted in Fig. 3. The main observation is that there is a broadening of the complete spectrum when one increases $\lambda$. In Fig. 3(a) we see that the averaged smoothed density of states changes in a smooth way from (16) to (17). But in Fig. 3(b) and much more in Figs. 3(c) and 3(d), the two semicircular densities $n^{w}[\varepsilon-(\Delta / 2)]$ and $n^{w}[\varepsilon+(\Delta / 2)]$ seem to repel each other as $\lambda$ increases. This is due to the fact that the levels of a given semicircular density do not interact with each other but only interact with the levels of the other semicircular density. This is a consequence of the nondiagonal form of the coupling. Therefore, having in mind the perturbative expression of the energies [see Eq. (C6) of Appendix $\mathrm{C}]$, one understands that when $\Delta$ is nonzero, the eigenvalues that are repelling each other with the most efficiency are the ones closest to the center of the total spectrum.

\section{B. Eigenvalue diagrams}

The global effect of the increase of $\lambda$ on the eigenvalues has been studied with the average smoothed density of states. But in order to have an idea of what happens on a finer
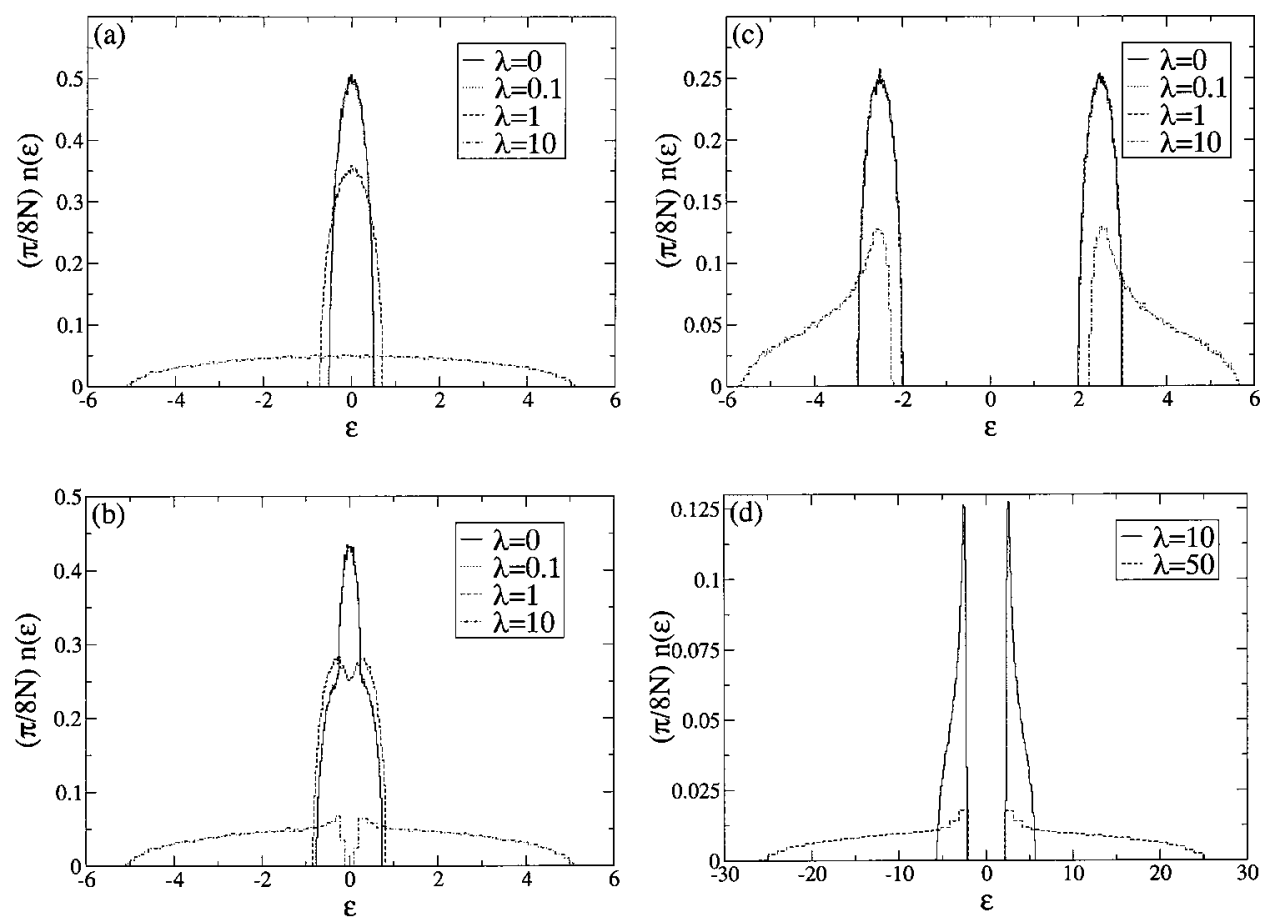

FIG. 3. Total smoothed averaged density of states obtained numerically for different values of $\Delta$ and $\lambda$. (a) corresponds to $\Delta=0.01$, (b) to $\Delta=0.5$, and (c) and (d) to $\Delta=5$. For all of them $N=500$ and $\chi=50$. Notice that the $\lambda=0$ and the $\lambda=0.1$ curves are not distinguishable. 

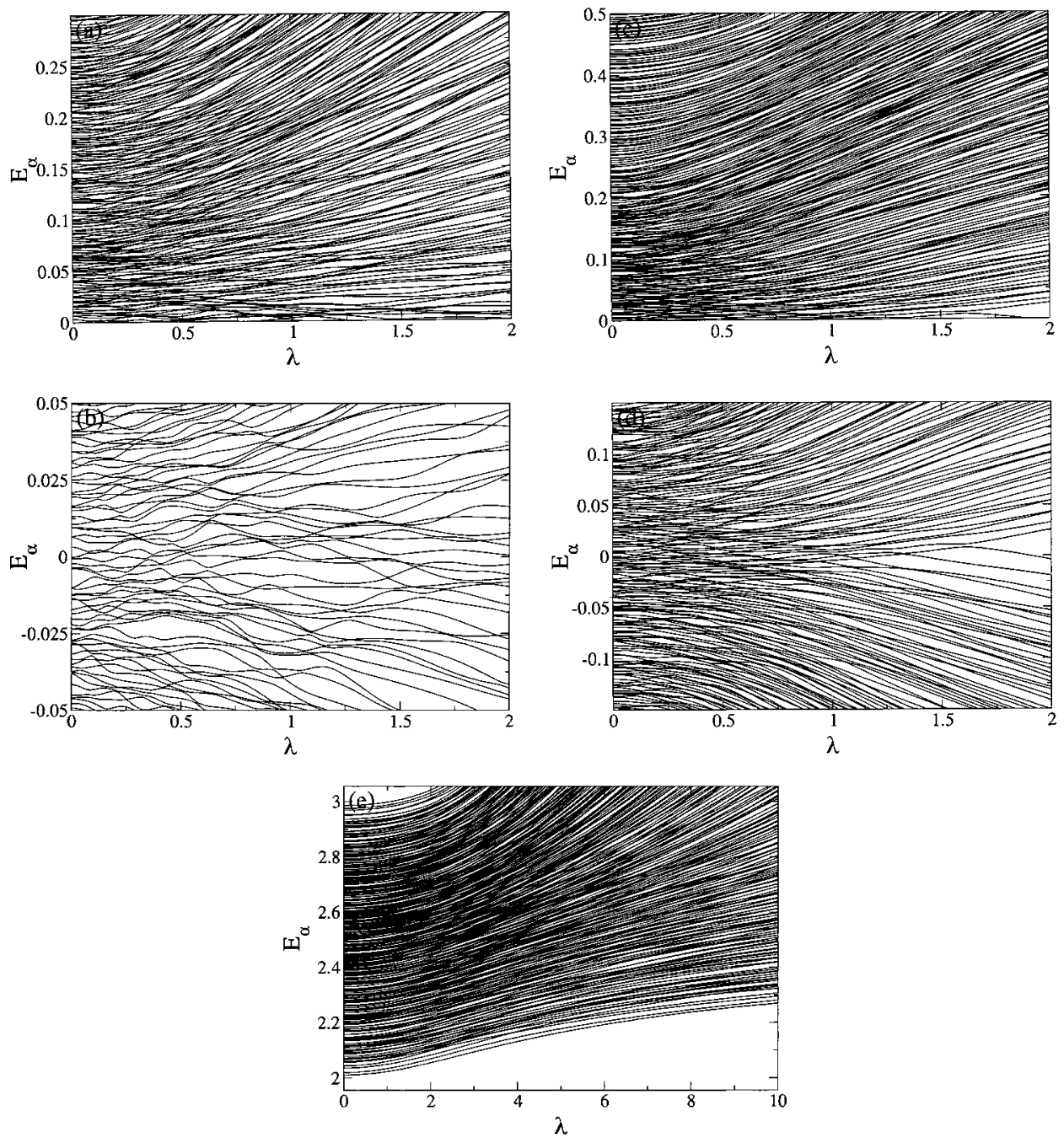

FIG. 4. Different parts of the eigenvalue diagrams with $N$ $=500$, corresponding to (a) and (b) $\Delta=0.01$, (c) and (d) $\Delta=0.5$, and (e) $\Delta=5$. They represent the eigenvalues of the total Hamiltonian (14) as a function of $\lambda$. energy scale inside the spectrum, it is interesting to individually follow each eigenvalue $E_{\alpha}$ as a function of $\lambda$ on an eigenvalue diagram.

The first thing to note (see Fig. 4) is that the increase of the coupling induces a repulsion between the eigenvalues. This has as a result the broadening of the total spectrum as we already noticed on the smoothed averaged density of states. If one looks closer inside the fine structure of the eigenvalue spectrum, we see that there is no crossing between the eigenvalues. This is a consequence of the fact that there is no symmetry in the total system. Therefore, the noncrossing rule is always working. Each time two eigenvalues come close to each other, they repel each other and create an avoided crossing. One can notice that there is a large number of avoided crossings inside the region where the two semicircular densities $n^{w}[\varepsilon-(\Delta / 2)]$ and $n^{w}[\varepsilon+(\Delta / 2)]$ overlap [see Fig. 2 in order to visualize the overlapping zone that extends from $(\Delta / 2)-1$ to $1-(\Delta / 2)]$. But outside this overlapping zone, there are very weak avoided crossings (and of course no crossing) and all the eigenvalues appear to move in a regular and smooth way. The regions seen in Figs. 4(a), 4(b), and 4(d) are inside the overlapping zone and the one in Fig. 4(e) is outside. In Fig. 4(c) the lower part of the diagram is inside the overlapping zone and the upper part is outside.
This phenomenon is due to the fact that the eigenvalues of a given semicircular density do not interact with the eigenvalues of their own semicircular density but only with those of the other semicircular density. It is the consequence of the nondiagonal nature of the coupling in the spin degrees of freedom. When the coupling becomes large enough $(\lambda>1)$, the avoided crossings also disappear inside the overlapping zone. Around $\lambda=1$ and inside the overlapping zone, there is a smooth transition from an avoided-crossing regime that gives rise to a turbulent and complex $\lambda$ evolution to another regime without much interaction between the eigenvalues that gives rise to a smooth $\lambda$ evolution.

\section{Spacing distribution}

The eigenvalue diagrams only give us a qualitative understanding of the fine energy structure of the spectrum. For a more quantitative study, it is interesting to look at the spacing distribution of the spectrum.

When $\lambda=0$, each semicircular distribution, corresponding to a different system level, has a Wignerian level spacing distribution,

$$
P^{w}(s)=\frac{\pi}{2} s e^{-(\pi / 4) s^{2}}
$$



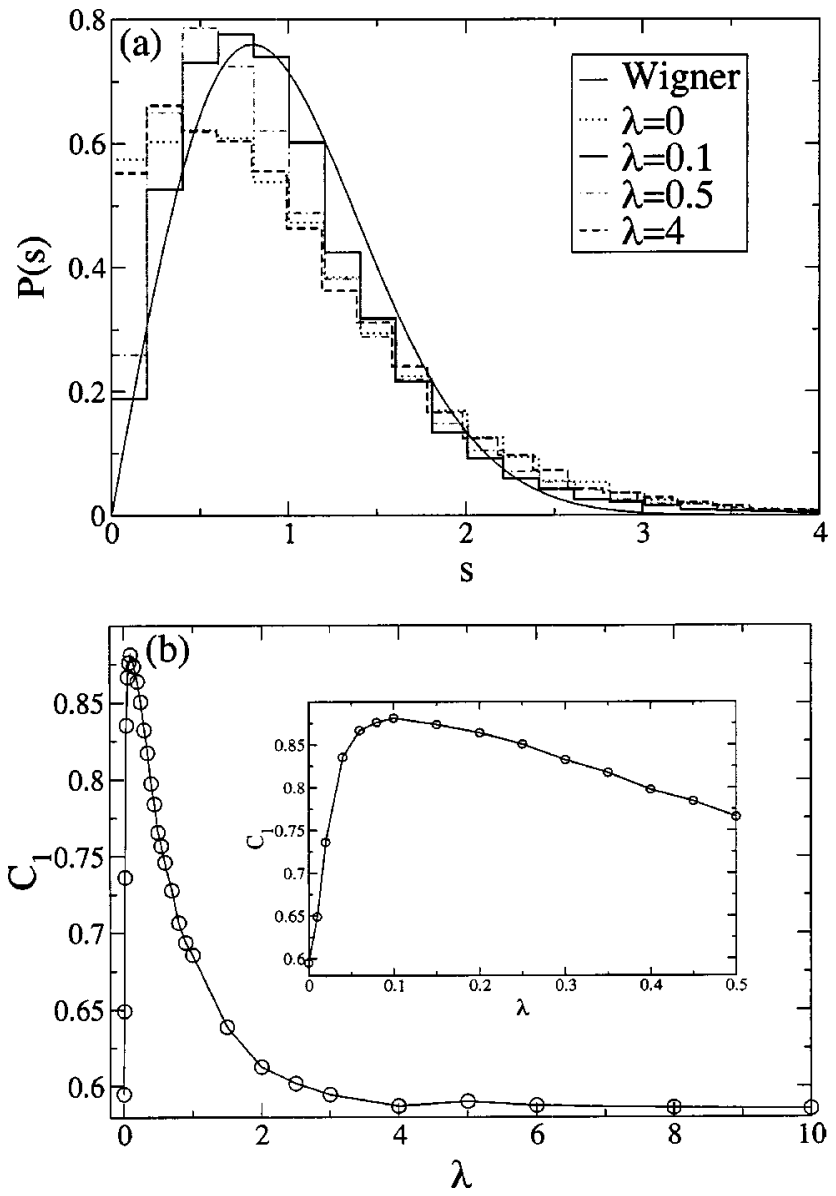

FIG. 5. (a) Spacing distribution for different values of the coupling parameter $\lambda$. (b) Fitted coefficient $C_{1}$ of Eq. (20). The closer is $C_{1}$ from unity, the closer is the spacing distribution to the Wigner spacing distribution. In the two figures, $\Delta=0.01, N=500$, and $\chi=50$.

But the total spectrum is made by the superposition, with a shift $\Delta$, of two of such semicircular distributions. The shift creates a Poissonian component to the total spacing distribution in the overlapping zone $[(\Delta / 2)-1$ to $-(\Delta / 2)+1]$. A Poissonian distribution is given by

$$
P^{p}(s)=e^{-s}
$$

Therefore, we choose to fit the total spacing distribution by the mixture

$$
P^{f i t}(s)=C_{1} P^{w}(s)+\left(1-C_{1}\right) P^{p}(s) .
$$

This choice of the form of the fit is empirical but reasonable because the correlation coefficient of the fit is always close to one (between 0.963 and 0.982).

We computed the spacing distribution and made the fit (20) in order to compute the mixing coefficient $C_{1}$ for different values of $\lambda$ in the case where the overlapping zone covers almost the whole spectrum $(\Delta \ll 1)$. The results are plotted in Fig. 5. We see that there is a specific region of $\lambda$ values where the total spacing distribution is close to a pure Wignerian one. This region corresponds to the situation where $\lambda^{2} N=O(1)$, i.e., when the typical intensity of the

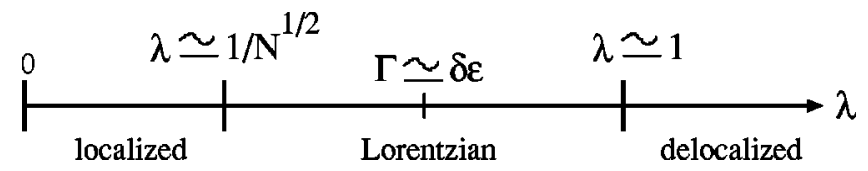

FIG. 6. Diagram of the different regimes as a function of the coupling parameter $\lambda$.

interaction between the nonperturbed levels is $O\left(\lambda^{2}\right)$ (since the first nonzero correction in perturbation theory is of second order due to the nondiagonal coupling in our model), becomes of the order of the mean level spacing $O(1 / N)$ in the total system. In this region the level repulsion is maximal and effective among almost all the states.

In the limit $\lambda \rightarrow \infty$ the spectrum is again the superposition of two semicircular distributions, one for the $E_{1 \eta}$, s and one for the $E_{-1 \eta}$ 's according to Eq. (13). As a consequence, one gets a Poissonian type of spacing distribution.

The other $\Delta$ cases will have a Wignerian spacing distribution outside the overlapping zone and a mixed one (like for the case $\Delta \ll 1)$ inside. This can be visually seen on the eigenvalue diagram that we studied before.

\section{The shape of the eigenstates (SOE)}

We want now to have some information about the eigenstates of the total system inside the overlapping zone of the two semicircular densities. Following [6], we define the quantity

$$
\xi\left(\varepsilon, \varepsilon^{0}\right)=\sum_{\alpha, n}|\langle\alpha \mid n\rangle|^{2} \delta\left(E_{\alpha}-\varepsilon\right) \delta\left(E_{n}-\varepsilon^{0}\right)
$$

If we fix $\varepsilon^{0}$ and study $\xi\left(\varepsilon, \varepsilon^{0}\right)$ as a function of $\varepsilon$, we will call it the local density of states (LDOS). If we fix $\varepsilon$ and study $\xi\left(\varepsilon, \varepsilon^{0}\right)$ as a function of $\varepsilon^{0}$, we will call it the SOE (shape of the eigenstates).

We here focus on the SOE. The SOE tells us how close a perturbed eigenstate $(\lambda \neq 0)$ at energy $\varepsilon$ is from the nonperturbed eigenstate $(\lambda=0)$ at energy $\varepsilon^{0}$. If the SOE is a very narrow function centered around $\varepsilon=\varepsilon^{0}$, the concept of a nonperturbed eigenstate is still useful. This regime corresponds to very small coupling, for which the interaction intensity is lower than the mean level spacing $0<\lambda^{2} N \lesssim 1$, and will be called to the localized regime. In the limit $N \rightarrow \infty$ this regime disappears. If one increases the coupling, the interaction intensity between the nonperturbed states begins to be larger than the mean level spacing between the states: $\lambda^{2} N$ $>1$. The nonperturbed levels start to be "mixed" by the interaction and the SOE starts then to have a Lorentzian shape with a finite width $\Gamma$, centered around $\varepsilon=\varepsilon^{0}$. This regime is called the Lorentzian regime. If one further increases the coupling parameter, the SOE begins to spread over almost the whole spectrum. This regime is called the delocalized regime.

In the banded random matrix model of [6], the regimes are classified according to a different terminology and there is an additional regime corresponding to a spreading that goes beyond the energy range where the coupling acts (due 




FIG. 7. Schematic representation of the different regimes in the plane of the reduced parameter $\lambda$ and $N$. The Lorentzian 1 regime corresponds to $\Gamma \leqq \delta \epsilon$ and the Lorentzian 2 regime to $\Gamma>\delta \epsilon$.

to the finite coupling range of the banded matrices). The motivation for our change of terminology will become clear in the study of the dynamics.

The Lorentzian regime can be separated into two parts. For small coupling, the width of the Lorentzian $\Gamma$ is smaller or of the order of magnitude of the typical energy scale of variation of the averaged smoothed density of state of the environment $\delta \epsilon: n(\epsilon+\delta \epsilon) \approx n(\epsilon)$. For larger coupling, the Lorentzian width extends on an energy scale larger than the typical energy scale of variation of the density of states of the environment. Therefore, one has $\Gamma \lessgtr \delta \epsilon$ in the former case and $\Gamma>\delta \epsilon$ in the latter case. The different regimes are represented in Figs. 6 and 7.

To represent the SOE of the spin-GORM model, we discretize the energy axis in small cells of the order of the mean level spacing $1 / N$ and we average the SOE over $\chi$ realizations of the random matrix ensemble. We see in Fig. 8(a) the typical shape of the SOE going from the perturbative regime $(\lambda=0.01,0.05)$ to the beginning of the Lorentzian one $(\lambda$ $=0.1$ ). In Fig. 8(b) we see the SOE across the Lorentzian regime $(0.2 \leqslant \lambda \leqslant 0.8)$. We also see the delocalized regime, when $\lambda \rightarrow \infty$ and the SOE gets completely flat $(\lambda=10)$. Figure 8 (c) shows the width of the Lorentzian from a fit made on the SOE curve. The correlation coefficient of the fit helps us to determine the region of the Lorentzian regime where the SOE is very well fitted by a Lorentzian. It has been verified that the width of the Lorentzian is independent of $N$ in the Lorentzian regime. Figure 8(d) (log-log) shows that the width of the Lorentzian has a power-law dependence in the coupling parameter close to two in the Lorentzian regime.

\section{E. Asymptotic transition probability kernel (ATPK)}

An interesting quantity, which is close to the SOE, but which has a nice physical interpretation, is the asymptotic transition probability kernel (ATPK). The transition probability kernel (TPK) gives the probability at time $t$ to be in the level $|n\rangle$ if starting from $\hat{\rho}(0)$. It is defined as

$$
\Pi_{t}(n \mid \rho(0))=\langle n| e^{-i \hat{H}_{\text {tot }} \hat{\rho}(0) e^{i \hat{H}_{\text {tot }} t}|n\rangle}
$$

The ATPK is the time average of the TPK

$$
\begin{aligned}
\Pi_{\infty}(n \mid \rho(0)) & =\lim _{T \rightarrow \infty} \frac{1}{T} \int_{0}^{T} d t \Pi_{t}(n \mid \rho(0)) \\
& =\sum_{\alpha}|\langle\alpha \mid n\rangle|^{2}\langle\alpha|\hat{\rho}(0)| \alpha\rangle .
\end{aligned}
$$

The distribution of the ATPK in energy is given by
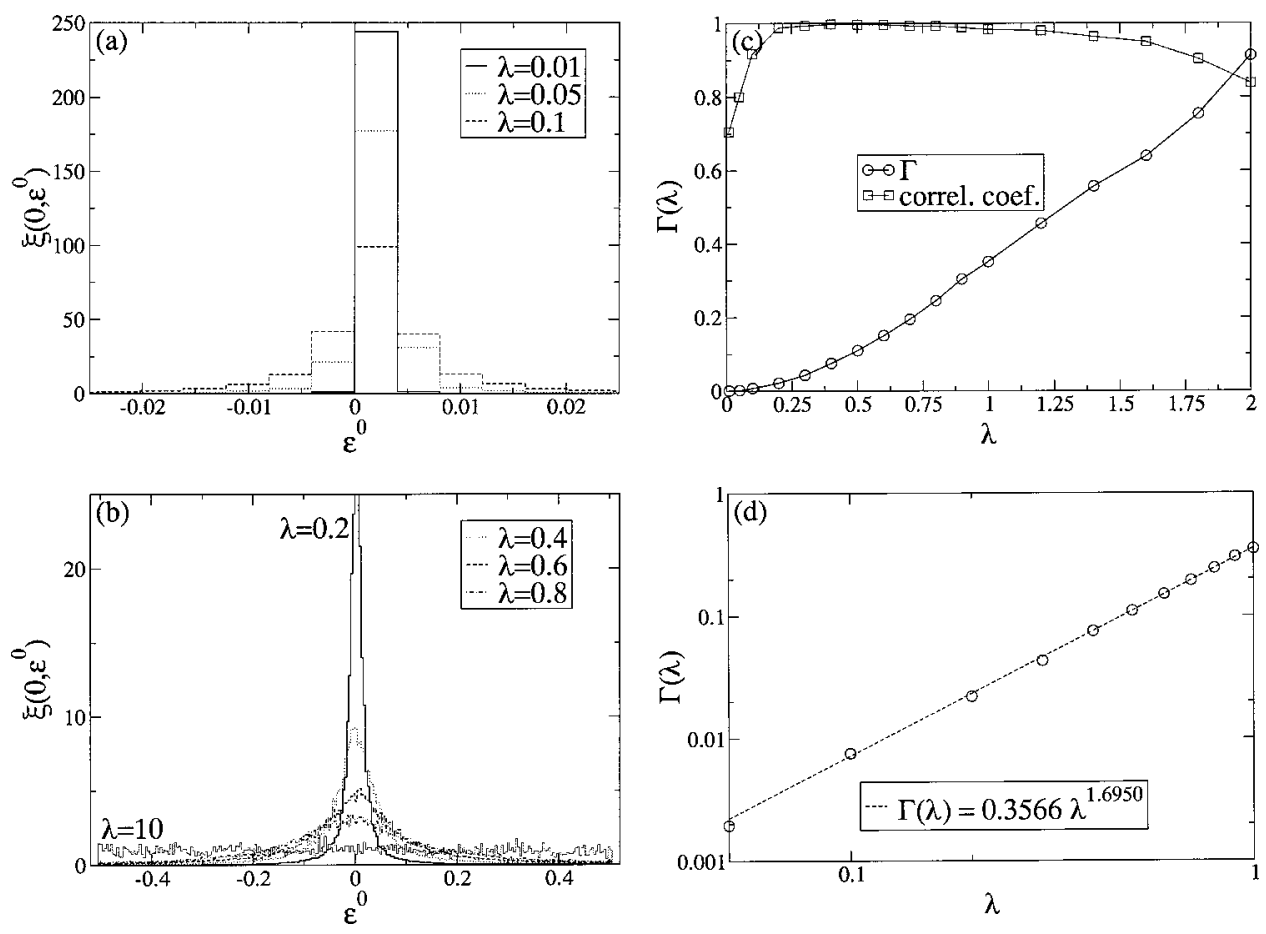

FIG. 8. (a) SOE in the perturbative regime. (b) SOE in the Lorentzian regime. (c) The width and the correlation coefficient of the fit of the SOE by a Lorentzian. (d) Power-law dependence of the width of the Lorentzian SOE in the coupling parameter. In all the figures: $\Delta=0.01, N=500, \chi=50$, and $\varepsilon=0$. 


$$
\Pi_{\infty}\left(\varepsilon^{0} \mid \rho(0)\right)=\sum_{n} \Pi_{\infty}(n \mid \rho(0)) \delta\left(E_{n}-\varepsilon^{0}\right) .
$$

The ATPK has an intuitive physical interpretation. It represents the probability after a very long time to end up in a nonperturbed state $|n\rangle$, having started from the initial condition $\hat{\rho}(0)$. The ATPK is the convolution of the SOE

$$
\begin{aligned}
\Pi_{\infty}\left(\varepsilon^{0} \mid \varepsilon^{0^{\prime}}\right)= & \sum_{n, m} \sum_{\alpha}|\langle\alpha \mid n\rangle|^{2}|\langle\alpha \mid m\rangle|^{2} \\
& \times \delta\left(E_{n}-\varepsilon^{0}\right) \delta\left(E_{m}-\varepsilon^{0^{\prime}}\right),
\end{aligned}
$$

with $\rho(0)=\Sigma_{m}|m\rangle\langle m| \delta\left(E_{m}-\varepsilon^{0^{\prime}}\right)$.

Because we are interested in a random matrix model and for the purpose of studying the dynamics, we will perform averages of two different kinds. The first kind of average is a microcanonical average over states belonging to the same given energy shell of width $\delta \varepsilon$ for a given realization of the random matrix ensemble used in our total Hamiltonian. The second kind of average is an ensemble average over the $\chi$ different realizations of the random matrices ensemble. For the microcanonical average, a choice of the width of the energy shell $\delta \varepsilon$ has to be done in such a way that it is large enough to contains many levels (to get a good statistics) and small enough to be smaller than or equal to the typical energy scale of variation of the averaged smoothed density of states of the environment $\delta \epsilon$. Therefore, the adequate choice of the width of the energy shell corresponds to $1 / N<\delta \varepsilon$ $<\delta \epsilon$.

Different ATPK are depicted in Fig. 9 where there is no random matrix ensemble average $\chi=1$. In Fig. 9(a) the ATPK is a microcanonical average inside the energy shell at energy $\varepsilon^{0^{\prime}}$ and of width $\delta \varepsilon^{0^{\prime}}$. "Pin" denotes the total probability of staying inside the energy shell after a very long time. $N \lambda^{2}=1$ is on the border between the localized and the Lorentzian regime and $\lambda=1,10$ in the delocalized regime. We see that, until $N \lambda^{2}=1$, the main probability stays in the initial energy shell. But when the Lorentzian regime starts, the probability spreads over energies larger than $\delta \epsilon$ (here $\delta \epsilon$ $\approx \delta \varepsilon^{0^{\prime}}$ ). In Figs. 9(b) and 9(c) no average has been done. The initial condition is a nonperturbed pure state (corresponding to an energy close to zero) and one can see the individual probability of being on another nonperturbed state for the different regimes. We can see that in the localized regime the probability of staying on the initial state is much more important than the probability of leaving it. We also see that the Lorentzian regime starts when the initial state loses its privileged position containing the main probability and, therefore, when the neighboring levels begin to have an important fraction of the total probability.

\section{TIME EVOLUTION}

We now want to understand the time evolution of our model and more specifically the population dynamics of the system. The exact evolution of the total system is described by the von Neumann equation

$$
\dot{\hat{\rho}}(t)=-i\left[\hat{H}_{\mathrm{tot}}, \hat{\rho}(t)\right]
$$

where $\hat{H}_{\text {tot }}$ is given by Eq. (5). The system dynamics is obtained from Eq. (26) using the reduced density matrix $\hat{\rho}_{S}(t)=\operatorname{Tr}_{B} \hat{\rho}(t)$. The total system has a finite and constant energy. At initial time, the environment has a given fixed energy $\epsilon$ corresponding to a microcanonical distribution inside a energy shell centered at $\epsilon$ and of width $\delta \epsilon . \delta \varepsilon$ is chosen in such a way that it is large enough to contains many levels (to get a good statistics) and small enough to be smaller than or equal to the typical energy scale of variation of the averaged smoothed density of state of the environment $\delta \epsilon$. Therefore, the adequate choice for the width of the energy shell corresponds to $1 / N<\delta \varepsilon<\delta \epsilon$. The dynamics is then averaged over the $\chi$ realizations of the random matrix ensemble. In the following, we consider in detail the two different extreme cases of weak and strong couplings.

\section{A. The weak coupling regime $(\lambda \ll 1)$}

We derived in Ref. [17] a perturbative equation for the description of the evolution of a system (with a discrete spectrum) weakly interacting with its environment (with a quasicontinuous spectrum). This equation has been shown to be equivalent to the well-known Redfield equation $[10,18,19]$ when the typical energy scale of the system (typical energy spacing between the system levels) can be considered small compared to the typical environment energy scale $\delta \epsilon$ (typical energy scales on which the smoothed density of states of the environment varies). In the spin-GORM model, this condition means that $n(\epsilon+\Delta) \approx n(\epsilon)$.

We will not perform in this paper the detailed derivation of this equation and its application to the spin-GOE model. This has been done in Ref. [17]. We will simply recall the main ideas and results of this paper and apply them to the study of the dynamics of our model.

The main idea is to suppose that the total density matrix can be described at all time by a density matrix of the following form:

$$
\hat{\rho}(t)=\frac{1}{n\left(\hat{H}_{B}\right)} \sum_{s, s^{\prime}}|s\rangle\left\langle s^{\prime}\right| P_{s s^{\prime}}\left(\hat{H}_{B} ; t\right),
$$

where the environment density of states is

$$
n(\epsilon)=\operatorname{Tr}_{B} \delta\left(\epsilon-\hat{H}_{B}\right) .
$$

Doing this, we neglect the contribution to the dynamics coming from the coherence of the environment but we keep those of the system. Therefore, the total density matrix is diagonal in the environment degrees of freedom but not in the system ones. For comparison, let us recall that in the derivation of the well known Pauli equation, both types of coherences are neglected and the total density matrix is completely diagonal [20-22].

The matrix of elements $P_{s s^{\prime}}\left(\hat{H}_{B} ; t\right)$ is Hermitian,

$$
P_{s s^{\prime}}\left(\hat{H}_{B} ; t\right)=P_{s^{\prime} s}^{*}\left(\hat{H}_{B} ; t\right)
$$





FIG. 9. (a) ATPK from the localized regime, through the Lorentzian regime, to the delocalized regime. The ATPK is microcanonically averaged over the different initial conditions corresponding to the levels inside the energy shell centered at $\varepsilon^{0^{\prime}}=0$ with width $\delta \varepsilon^{0^{\prime}}=0.05$. "Pin" denotes the proportion of the ATPK that stays inside the initial energy shell after an infinite time. (b) and (c) ATPK for a single level as an initial condition, without any average. All the figures are obtained for very small system energy $\Delta=0.01$ with no random matrix ensemble average $\chi=1$ and for $N=500$.
The reduced density matrix of the system becomes

$$
\begin{aligned}
\hat{\rho}_{S}(t) & =\operatorname{Tr}_{B} \hat{\rho}(t) \\
& =\int d \epsilon \operatorname{Tr}_{B} \delta\left(\epsilon-\hat{H}_{B}\right) \hat{\rho}(t) \\
& =\sum_{s, s^{\prime}}|s\rangle\left\langle s^{\prime}\right| \int d \epsilon P_{s s^{\prime}}(\epsilon ; t) .
\end{aligned}
$$

We see that each element of the reduced density matrix of the system depends on the environment energy. This is fundamental in order to take into account the finite energy effects of the total system.

Using Eq. (27) and performing a perturbative expansion up to the second order in $\lambda$ on Eq. (26) (see Ref. [17]), one gets for the population dynamics the equation

$$
\begin{aligned}
\dot{P}_{s s}(\epsilon ; t)= & -2 \lambda^{2} \sum_{\bar{s}, \bar{s}^{\prime}}\left[+\left\langle s|\hat{S}| \bar{s}^{\prime}\right\rangle\left\langle\bar{s}^{\prime}|\hat{S}| \bar{s}\right\rangle P_{\bar{s} s}(\epsilon ; t)\right. \\
& \times \int d \epsilon^{\prime} F\left(\epsilon, \epsilon^{\prime}\right) n\left(\epsilon^{\prime}\right) \frac{\sin \left(E_{\bar{s}}-E_{\bar{s}^{\prime}}+\epsilon-\epsilon^{\prime}\right) \tau}{E_{\bar{s}}-E_{\bar{s}^{\prime}}+\epsilon-\epsilon^{\prime}} \\
& -\langle s|\hat{S}| \bar{s}\rangle\left\langle\bar{s}^{\prime}|\hat{S}| s\right\rangle n(\epsilon) \int d \epsilon^{\prime} F\left(\epsilon, \epsilon^{\prime}\right) \\
& \left.\times P_{\overline{s \bar{s}^{\prime}}}\left(\epsilon^{\prime} ; t\right) \frac{\sin \left(E_{s}-E_{\bar{s}^{\prime}}+\epsilon-\epsilon^{\prime}\right) \tau}{E_{s}-E_{\bar{s}^{\prime}}+\epsilon-\epsilon^{\prime}}\right]
\end{aligned}
$$

where $F\left(\epsilon, \epsilon^{\prime}\right)=$ " $\left|\left\langle\epsilon|\hat{B}| \epsilon^{\prime}\right\rangle\right|^{2}$," where the quotes denote a smoothening over a dense spectrum of eigenvalues around $\epsilon$ and $\epsilon^{\prime}$. We see that the probability $\dot{P}_{\bar{s} s}(\epsilon ; t)$, if initially concentrated in a given energy shell, can spread in energy under the dynamics. This is a typical non-Markovian effect happening on a short-time scale. It is due to the presence in the equation of the energy integral and of the $\sin (\xi \tau) / \xi$ function that has a finite width in energy at short time. This equation is non-Markovian in the sense that the coefficients of the differential evolution equation are time dependent and that this time dependence can be neglected on long-time scales (performing the Markovian approximation) when the environment has a faster dynamics (i.e., typically a larger energy scale) than the system.

The Markovian approximation consists of taking the infinite-time limit of the time-dependent coefficients using the property $\lim _{\tau \rightarrow \infty}[\sin (\xi \tau) / \xi]=\pi \delta(\xi)$. This approximation is justified if the contribution of the $\xi=0$ value (if it exists) has the main and almost unique contribution to the energy integral. If one further neglects the contributions of the coherence to the population evolution (this is automatically satisfied for the spin-GORM model because of the nondiagonal coupling), one gets a Pauli-type equation [20-22] 


$$
\begin{aligned}
\dot{P}_{s s}(\epsilon ; t)= & -2 \pi \lambda^{2} \sum_{s^{\prime} \neq s}\left|\left\langle s|\hat{S}| s^{\prime}\right\rangle\right|^{2} F\left(\epsilon, E_{s}-E_{s^{\prime}}+\epsilon\right) \\
& \times n\left(E_{s}-E_{s^{\prime}}+\epsilon\right) P_{s s}(\epsilon ; t) \\
& +2 \pi \lambda^{2} \sum_{s^{\prime} \neq s}\left|\left\langle s|\hat{S}| s^{\prime}\right\rangle\right|^{2} F\left(\epsilon, E_{s}-E_{s^{\prime}}+\epsilon\right) \\
& \times n(\epsilon) P_{s^{\prime} s^{\prime}}\left(E_{s}-E_{s^{\prime}}+\epsilon ; t\right) .
\end{aligned}
$$

We see that the Markovian approximation strictly keeps the dynamics of the total system inside an energy shell. Starting with the probability located on a given energy shell, the dynamics will preserve the probability inside this shell. But of course, the probability of the different states inside the shell are varying. The dynamics described by this equation can be seen as a random walk between nonperturbed states of the total system belonging to the same energy shell with transition rates between these states given by the Fermi golden rule.

We now apply our equation to the spin-GORM model in order to study of the population evolution through $\hat{\sigma}_{z}$ (the difference between the probability of being in the upper state of the system minus the probability of being in the lower one). Doing this, we suppose that the environment is quasicontinuous $N \rightarrow \infty$ and that the random matrix ensemble average has been performed $\chi \rightarrow \infty$. For the non-Markovian equation (31), one gets

$$
\left\langle\hat{\sigma}_{z}\right\rangle^{N M}(t)=\int d \epsilon^{\prime}\left[P_{++}\left(\epsilon^{\prime} ; t\right)-P_{--}\left(\epsilon^{\prime}+\Delta ; t\right)\right],
$$

where

$$
\begin{aligned}
\dot{P}_{ \pm \pm}(\epsilon ; t)= & \frac{\lambda^{2}}{\pi} \int_{-1 / 2}^{+1 / 2} d \epsilon^{\prime} \frac{\sin \left( \pm \Delta+\epsilon-\epsilon^{\prime}\right) t}{\left( \pm \Delta+\epsilon-\epsilon^{\prime}\right)} \\
& \times\left[P_{\mp \mp}\left(\epsilon^{\prime} ; t\right) \sqrt{\frac{1}{4}-\epsilon^{2}}\right. \\
& \left.-P_{ \pm \pm}(\epsilon ; t) \sqrt{\frac{1}{4}-\epsilon^{\prime 2}}\right] .
\end{aligned}
$$

In the Markovian limit, Eq. (33) becomes

$$
\left\langle\hat{\sigma}_{z}\right\rangle^{M}(t)=P_{++}(\epsilon ; t)-P_{--}(\epsilon+\Delta ; t),
$$

which obeys a Pauli-type equation so that we get

$$
\left\langle\hat{\sigma}_{z}\right\rangle^{M}(t)=\left\langle\hat{\sigma}_{z}\right\rangle_{\infty}^{M}+\left[\left\langle\hat{\sigma}_{z}\right\rangle^{M}(0)-\left\langle\hat{\sigma}_{z}\right\rangle_{\infty}^{M}\right] e^{-\gamma t},
$$

where the equilibrium value of the populations is given by

$$
\left\langle\hat{\sigma}_{z}\right\rangle_{\infty}^{M}=\frac{\sqrt{\frac{1}{4}-(\epsilon)^{2}}-\sqrt{\frac{1}{4}-(\epsilon+\Delta)^{2}}}{\sqrt{\frac{1}{4}-(\epsilon)^{2}}+\sqrt{\frac{1}{4}-(\epsilon+\Delta)^{2}}}
$$

and the relaxation rate by

$$
\gamma=\lambda^{2}\left(\sqrt{\frac{1}{4}-(\epsilon)^{2}}+\sqrt{\frac{1}{4}-(\epsilon+\Delta)^{2}}\right),
$$

where we adopted the convection that $\sqrt{x}=0$ if $x<0$.

In the case of the spin-GORM model, the transition probability between states belonging to the same total energy shell only depends on the smoothed density of states. This is due to the fact that the environment coupling elements between the environment nonperturbed states are randomly distributed because the environment coupling operator is a random matrix. This has the consequence that the equilibrium value of the populations (37) corresponds to a microcanonical distribution probability of the states belonging to the total energy shell independently of the initial distribution of these states inside this energy shell.

We notice that, in the general case, the Markovian assumption made on our equation does not directly give a Pauli-type equation which is an equation for the populations only. To get a Pauli equation, the further approximation, which consists of neglecting the contribution of the coherences to the population dynamics, has to be done. For the particular case of the spin-GORM model, this further approximation is not necessary because it is automatically satisfied.

\section{B. The strong coupling regime $(\lambda \gg 1)$}

We are now interested in describing the dynamical regime where the coupling parameter $\lambda$ is very large and, therefore, dominant in front of 1 and $\Delta$. We will again suppose that the environment is continuous $N \rightarrow \infty$ and that the random matrix ensemble average has been performed $\chi \rightarrow \infty$.

The population dynamics of the system is given by

$$
\begin{aligned}
\left\langle\hat{\sigma}_{z}\right\rangle(t)= & \operatorname{Tr} \hat{\rho}(t) \hat{\sigma}_{z}=\operatorname{Tr} e^{i\left[(\Delta / 2) \hat{\sigma}_{z}+\hat{H}_{B}+\lambda \hat{\sigma}_{x} \hat{B}\right] t} \hat{\rho}(0) \\
& \times e^{-i\left[(\Delta / 2) \hat{\sigma}_{z}+\hat{H}_{B}+\lambda \hat{\sigma}_{x} \hat{B}\right] t} \hat{\sigma}_{z} .
\end{aligned}
$$

Using the following unitary transformation acting on the spin degrees of freedom:

$$
\hat{U}=\left(\begin{array}{cc}
\frac{1}{\sqrt{2}} & \frac{1}{\sqrt{2}} \\
\frac{1}{\sqrt{2}} & -\frac{1}{\sqrt{2}}
\end{array}\right)
$$

we get

$$
\begin{aligned}
\left\langle\hat{\sigma}_{z}\right\rangle(t)= & \operatorname{Tr} e^{i\left[(\Delta / 2) \hat{\sigma}_{x}+\hat{H}_{B}+\lambda \hat{\sigma}_{z} \hat{B}\right] t} \hat{U}^{\dagger} \hat{\rho}_{S}(0) \hat{U} \\
& \times \hat{\rho}_{B}(0) e^{-i\left[(\Delta / 2) \hat{\sigma}_{x}+\hat{H}_{B}+\lambda \hat{\sigma}_{z} \hat{B}\right] t} \hat{\sigma}_{x} \\
= & \operatorname{Tr} e^{i \lambda\left[(1 / \lambda)(\Delta / 2) \hat{\sigma}_{x}+(1 / \lambda) \hat{H}_{B}+\hat{\sigma}_{z} \hat{B}\right] t} \hat{U}^{\dagger} \hat{\rho}_{S}(0) \hat{U} \\
& \times \hat{\rho}_{B}(0) e^{-i \lambda\left[(1 / \lambda)(\Delta / 2) \hat{\sigma}_{x}+(1 / \lambda) \hat{H}_{B}+\hat{\sigma}_{z} \hat{\sigma}\right] t} \hat{\sigma}_{x}
\end{aligned}
$$


Using the following perturbative expansion of the evolution operator to order zero in $1 / \lambda$ :

$$
e^{-i \lambda\left[(1 / \lambda)(\Delta / 2) \hat{\sigma}_{x}+(1 / \lambda) \hat{H}_{B}+\hat{\sigma}_{z} \hat{B}\right] t} \stackrel{1 / \lambda \rightarrow 0}{=} e^{-i \lambda t \hat{\sigma}_{z} \hat{B}}+O\left(\frac{1}{\lambda}\right),
$$

we get

$$
\left\langle\hat{\sigma}_{z}\right\rangle(t) \stackrel{1 / \lambda \rightarrow 0}{=} \operatorname{Tr} e^{i \lambda t \hat{\sigma}_{z} \hat{B}} \hat{U}^{\dagger} \hat{\rho}_{S}(0) \hat{U} \hat{\rho}_{B}(0) e^{-i \lambda t \hat{\sigma}_{z} \hat{B}}+O\left(\frac{1}{\lambda}\right) .
$$

Using the following notation:

$$
\hat{\sigma}_{z} \hat{B}|\kappa \eta\rangle=\hat{\sigma}_{z}|\kappa\rangle \otimes \hat{B}|\eta\rangle=\kappa E_{\eta}|\kappa \eta\rangle
$$

and

$$
\hat{H}_{B}|b\rangle=E_{b}^{B}|b\rangle,
$$

we find that

$$
\begin{aligned}
\left\langle\hat{\sigma}_{z}\right\rangle(t) \stackrel{1 / \lambda \rightarrow 0}{=} & \sum_{\kappa, \eta} e^{i \lambda t \kappa E} \eta\left\langle\eta\left|\hat{\rho}_{B}(0)\right| \eta\right\rangle\left\langle\kappa\left|\hat{U}^{\dagger} \hat{\rho}_{S}(0) \hat{U}\right|-\kappa\right\rangle \\
& \times e^{i \lambda t \kappa E_{\eta}}+O\left(\frac{1}{\lambda}\right) .
\end{aligned}
$$

Because $\hat{\rho}_{B}$ is diagonal in the basis that diagonalizes $\hat{H}_{B}$, we have

$$
\left\langle\eta\left|\hat{\rho}_{B}(0)\right| \eta\right\rangle=\sum_{b}|\langle\eta \mid b\rangle|^{2}\left\langle b\left|\hat{\rho}_{B}\right| b\right\rangle .
$$

If we perform an ensemble average over different realizations of $\hat{H}_{B}$ and use the random matrix eigenvectors statistics [14-16], we find

$$
\begin{aligned}
\overline{\left\langle\eta\left|\hat{\rho}_{B}(0)\right| \eta\right\rangle^{\left(\hat{H}_{B}\right)}} & =\sum_{b} \overline{|\langle\eta \mid b\rangle|^{2}}\left(\hat{H}_{B}\right) \overline{\left\langle b\left|\hat{\rho}_{B}\right| b\right\rangle^{(}}\left(\hat{H}_{B}\right) \\
& =\sum_{b} \frac{2}{N} \overline{\left\langle b\left|\hat{\rho}_{B}\right| b\right\rangle^{\left(\hat{H}_{B}\right)}} \\
& =\frac{2}{N} .
\end{aligned}
$$

Equation (46) therefore becomes

$$
\begin{aligned}
\overline{\left\langle\hat{\sigma}_{z}\right\rangle(t)^{\left(\hat{H}_{B}\right)}} \stackrel{1 / \lambda \rightarrow 0}{=} & \frac{2}{N} \sum_{\kappa, \eta} e^{2 i \lambda t \kappa E} \eta\left\langle\kappa\left|\hat{U}^{\dagger} \hat{\rho}_{S}(0) \hat{U}\right|-\kappa\right\rangle \\
= & \frac{2}{N}\left[\left\langle 1\left|\hat{U}^{\dagger} \hat{\rho}_{S}(0) \hat{U}\right|-1\right\rangle \sum_{\eta} e^{2 i \lambda t E_{\eta}}\right. \\
& \left.+\left\langle-1\left|\hat{U}^{\dagger} \hat{\rho}_{S}(0) \hat{U}\right| 1\right\rangle \sum_{\eta} e^{-2 i \lambda t E_{\eta}}\right]+O\left(\frac{1}{\lambda}\right) .
\end{aligned}
$$

Performing now the following ensemble average over different realizations of $\hat{B}$ :

$$
\begin{aligned}
\overline{\sum_{\eta} e^{2 i \lambda t E_{\eta}}(\hat{B})} & =\int_{-1 / 2}^{+1 / 2} d \epsilon n(\epsilon) e^{2 i \lambda t \epsilon} \\
& =\frac{4 N}{\pi} \int_{-1 / 2}^{+1 / 2} d \epsilon \sqrt{\frac{1}{4}-\epsilon^{2}} e^{2 i \lambda t \epsilon} \\
& =N \frac{J_{1}(\lambda t)}{\lambda t},
\end{aligned}
$$

we finally get

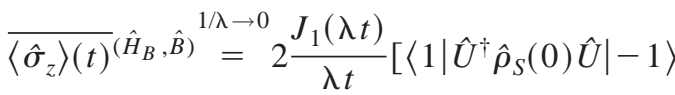

$$
\begin{aligned}
& \left.+\left\langle-1\left|\hat{U}^{\dagger} \hat{\rho}_{S}(0) \hat{U}\right| 1\right\rangle\right]+O\left(\frac{1}{\lambda^{2}}\right) .
\end{aligned}
$$

It is easy to show that the term of order $1 / \lambda$ is zero. This explains the fact that $O(1 / \lambda)$ has been replaced by $O\left(1 / \lambda^{2}\right)$.

Choosing as an initial condition

$$
\hat{\rho}_{S}(0)=\left(\begin{array}{ll}
1 & 0 \\
0 & 0
\end{array}\right),
$$

we get

$$
\overline{\left\langle\hat{\sigma}_{z}\right\rangle(t)^{\left(\hat{H}_{B}, \hat{B}\right)}} \stackrel{1 / \lambda \rightarrow 0}{=} 2 \frac{J_{1}(\lambda t)}{\lambda t}+O\left(\frac{1}{\lambda^{2}}\right) .
$$

We have found a well-defined behavior of the system dynamics when the coupling parameter $\lambda$ is so large that the coupling term can be considered to contribute alone to the dynamics.

\section{Numerical results}

We will now numerically study the validity of the approximated equation that we just derived in order to understand the dynamical evolution of our model.

In our numerical simulations, the initial condition of the system is always the upper state (52): $\left\langle\hat{\sigma}_{z}\right\rangle(0)=1$. The different parameter domains represented in Fig. 1 will play a fundamental role in our discussion of the dynamics.

We begin by the comparison between the results of the non-Markovian version (33) and (34) of our perturbative 

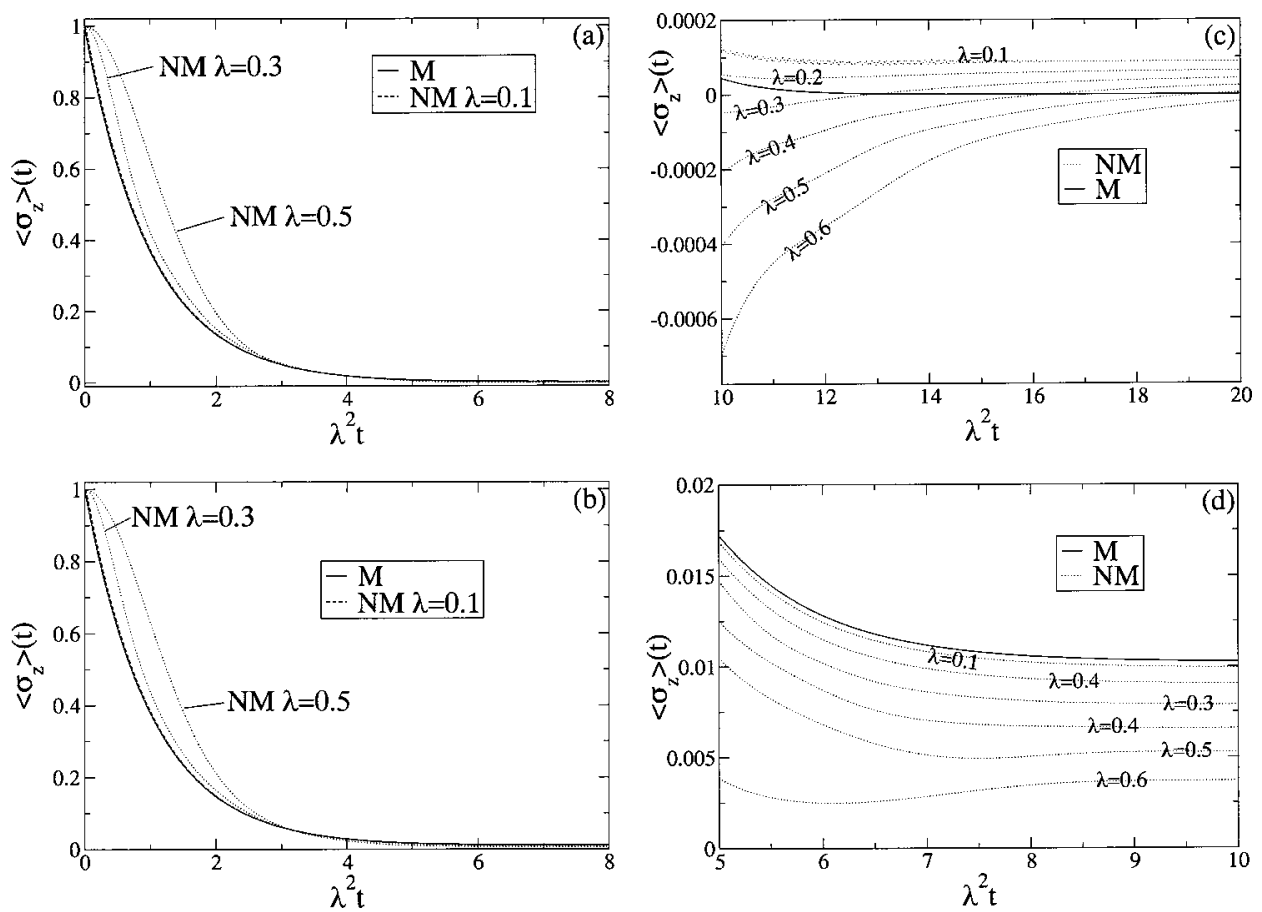

FIG. 10. Time evolution of the $z$ component of the spin: comparison between the Markovian (M) and non-Markovian (NM) versions of the perturbative equation for different values of the coupling parameter. In all the cases $\Delta=0.01$ and $\epsilon=0$.

equation and the results of the Markovian version (36)-(38) (of Pauli type) in order to understand better the consequences of the Markovian approximation. Figures 10(a) and 10(b) show the time evolution of $\left\langle\hat{\sigma}_{z}\right\rangle$ for both equations at different values of the coupling parameter. The time axis has been scaled by the coupling parameter $\left(\lambda^{2} t\right)$. This scaling is characteristic of the Lorentzian SOE regime. The values of $\lambda$ have of course to be reasonably small in order to remain consistent with the fact that these equations are obtained perturbatively. The time scale that we are observing is the global one: from the initial condition to the equilibrium. The char- acteristic energy of the system $(\Delta)$ is different in Figs. 10(a) and 10(b). But we are in both cases in domain A of the reduced parameter phase space (see Fig. 1). For such small values of $\Delta$, we see almost no difference between Figs. 10(a) and 10(b). The characteristic time scale of the environment is of order $2 \pi$, and is therefore in both cases much shorter than the system one. We are in situations where the Markovian approximation makes sense on times longer than $2 \pi$. Using the $\lambda^{2} t$ scaling, the Markovian equation is independent of $\lambda$. This is not the case for the non-Markovian equation. We see that the stronger $\lambda$ is, the larger the deviation is between both
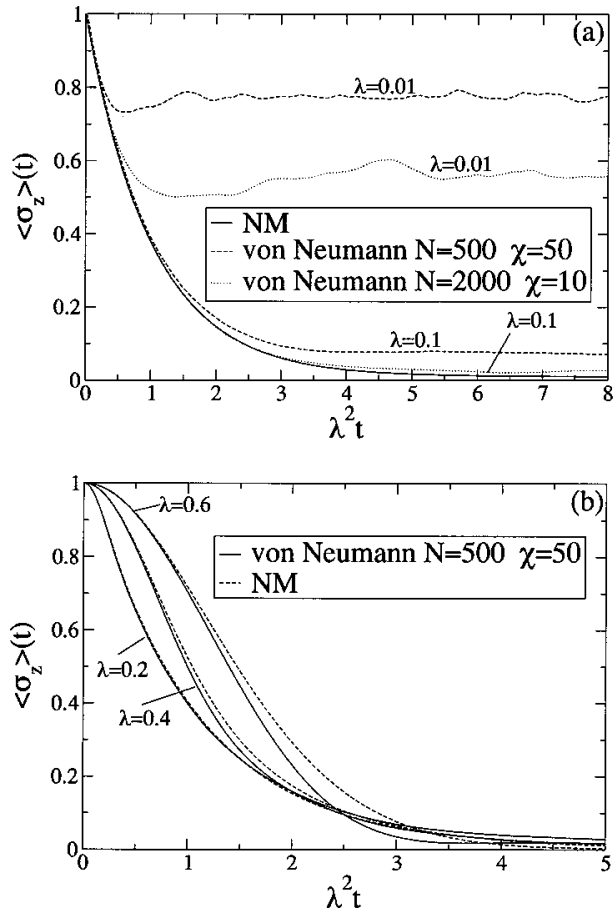


FIG. 11. Time evolution of the $z$ component of the spin. (a), (b), and (c) Comparison between the exact von Neumann equation and the non-Markovian (NM) version of the perturbative equation for different values of the coupling parameter. (a) and (b) correspond to $\Delta=0.1$ and (c) to $\Delta=0.5$ and $\lambda=0.1$. (d) Comparison between the exact von Neumann equation and the Bessel strong coupling result given by Eq. (53) for $\Delta=0.01$, $N=500$, and $\chi=50$. In all the figures $\chi=50$. 
equations. This is the consequence of the fact that the $\lambda^{2} t$ scaling that we use amplifies the non-Markovian short time behavior (that occurs on time of order of the characteristic time scale of the environment $2 \pi$ ) when $\lambda$ increases. Figures 10 (c) and 10(d) show us the long time behavior of the Markovian and non-Markovian versions of our equation. We see that the equilibrium value of $\left\langle\hat{\sigma}_{z}\right\rangle$ depends on $\lambda$ for the nonMarkovian equation. This is not the case for the Markovian equation. The differences between the equilibrium values are small. But, comparing Figs. 10(c) with plot 10(d), one notices that when $\Delta$ is larger, the differences are larger. This is a consequence of the error made on short-time dynamics using the Markovian approximation. Because this error is more important when $\lambda$ is large, the consequence on the long time dynamics is more important, even if globally small.

We now compare the non-Markovian version (33) and (34) of our perturbative equation with the exact von Neumann equation (26), staying in the parameter domain A. Figures 11(a) and 11(b) show the time evolution described by both equations at different $\lambda$ values using the $\lambda^{2} t$ time scaling. In Fig. 11(a) the Markovian and the non-Markovian versions of our equation are so close that we only plotted the second one. We see that the non-Markovian equation is valid not only for values of $\lambda$ below an upper bound, but also above a lower bound. When $\lambda$ is too small, the nonMarkovian version of our perturbative equation does not fit with the exact result. The exact initial dynamics is well reproduced by our equation, but the relaxation process to the equilibrium value is not reproduced. This is due to the discrete nature of the spectrum and therefore depends on the number $N$ of states and disappears in the limit $N \rightarrow \infty$. It corresponds to the border between the localized and Lorentzian regimes of the SOE. This is one of the main results of this paper. This phenomena is of course again related to the effect of the perturbation between the levels in the total spectrum that we already observed in the spacing distribution, in the SOE, and in the ATPK. $\lambda$ has to be large enough $\left(\lambda^{2}\right.$ $>1 / N)$ to "mix" the nonperturbed levels in order for all the states inside the total unperturbed energy shell to be mixed together. Remember that the equilibrium value of our Markovian perturbative equation is given by a microcanonical distribution in the total unperturbed energy shell [see Eq. (37)]. One also sees in Fig. 11(b) that our perturbative equation loses again its validity above a certain value of $\lambda$. It happens at value of $\lambda$ that cannot be considered as perturbative any more. It also corresponds to a value of the coupling parameter corresponding in the SOE to the transition in the Lorentzian regime when the width of the Lorentzian starts to be larger than the typical variation energy scale of the environment density of states.

In Fig. 11(c) we deal with the parameter domain B (see Fig. 1). We again compare the non-Markovian version of our perturbative equation with the exact dynamics given by the von Neumann equation. In this case, the system dynamics (of period $0.4 \pi$ ) is faster than the environment one (of period $2 \pi)$. We are in a highly non-Markovian situation. The Markovian version of our equation (that describes no evolution in this case) completely misses the observed behavior of damped oscillations. But the non-Markovian equation repro-

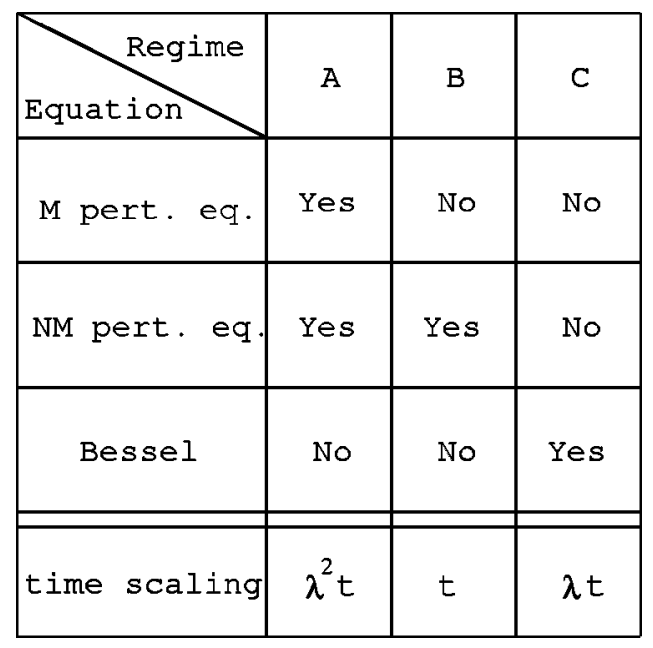

FIG. 12. Table giving the validity of the approximated equation and of the time scaling for the different parameter domains of the model. The parameter domains are defined in Fig. 1. "M pert. eq." means the Markovian verion of the perturbative equation (36), "NM pert. eq." refers to the Markovian version of the perturbative equation (33), and "Bessel" refers to the equation (53) obtained in the very strong coupling regime.

duces this behavior with a very high accuracy. The frequency of the oscillations corresponds to the system dynamics ones and the damping of these oscillations occur on a time scale corresponding to the environment characteristic time scale.

Finally, in Fig. 11(d) we are in the high coupling parameter domain $\mathrm{C}$ (see Fig. 1). We see that when $\lambda$ becomes large enough to make the coupling term dominant in the total Hamiltonian, the dynamics obey the Bessel behavior derived in Eq. (53). It is important to notice that this behavior scales in time according to $\lambda t$.

A summary of the validity of the different approximated equations [Markovian (36)-(38) and non-Markovian (33)(34) versions of our perturbative equation and the strong coupling Bessel equation (53)] and of the different scalings, depending on the regime that one considers, is represented in Fig. 12.

\section{Average versus individual realizations}

An interesting point is the comparison, for the system dynamics, between the averaged (random matrix ensemble averaged or microcanonically averaged) dynamics and the dynamics of an individual realization within the statistical ensembles. This latter corresponds to a dynamics generated by an initial condition that corresponds to a pure state and without any random-matrix average $(\chi=1)$.

We see in Figs. 13(c)-13(f) the dynamics of a system with small energy spacing $\Delta=0.1$ for different values of $\lambda^{2} N$. The solid line represents the random-matrix and microcanonically averaged dynamics. The dashed lines depict some of the individual members of the random-matrix ensemble corresponding to an initial pure state inside the total unperturbed energy shell. We see that the larger $\lambda^{2} N$ is, the closer the individual trajectories are from the averaged trajectory. We therefore have, when $\lambda^{2} N$ is large enough, that 

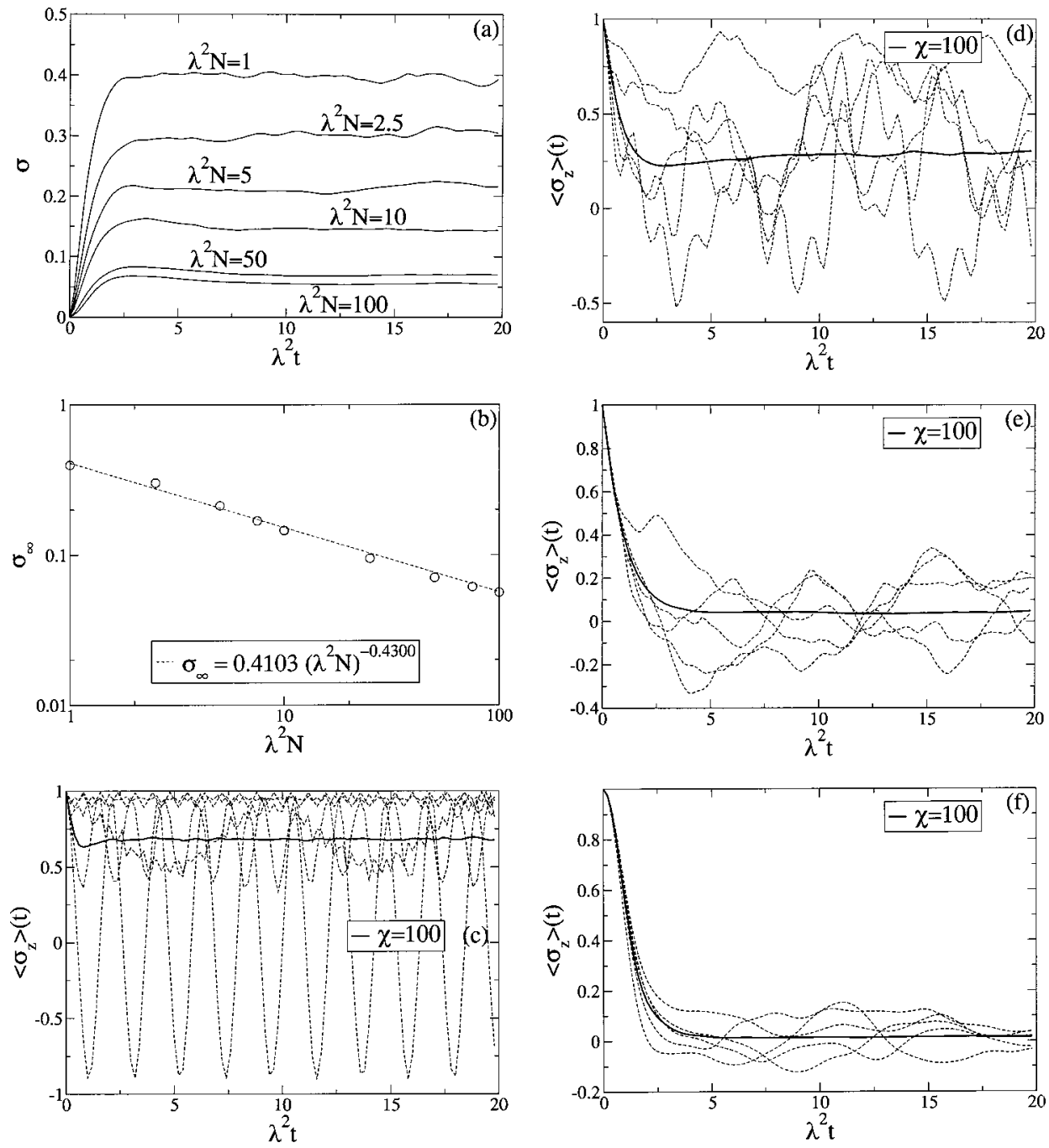

FIG. 13. In all the figures $\Delta=0.1, \quad N=500, \quad \epsilon=0$, and $\delta \epsilon$ $=0.05$. (a) Variance between individual trajectories and the averaged one $(\chi=100)$ as a function of time for different values of $\lambda^{2} N$. (b) Power-law dependence between the equilibrium value of this variance and $\lambda^{2} N$. (c)-(f) Individual trajectories of the ensemble (dashed lines) and the ensemble averaged trajectory (solid line). In (c) $\lambda^{2} N=0.1$, in (d) $\lambda^{2} N=1$, in (e) $\lambda^{2} N=10$, and in (f) $\lambda^{2} N=100$. the individual realizations are self-averaging in the randommatrix and microcanonical ensembles. In order to quantify this behavior, we plotted in Fig. 13(a) the variance between the individual trajectories and the averaged trajectory as a function of time for different values of $\lambda^{2} N$. We observe that this variance decreases as $\lambda^{2} N \rightarrow \infty$. In Fig. 13(b) we show that the asymptotic value of the variance decreases with a power-law dependence with respect to $\lambda^{2} N$.

We again see the relation with the SOE regimes. In the localized regime [Fig. 13(c)] the dynamics is governed by very different individual trajectories oscillating with a very few frequencies that differ from one individual trajectory to another. This is a consequence of the fact that the perturbed levels are still close to the nonperturbed ones and are only slightly affected by neighboring nonperturbed levels. In the Lorentzian regime [Figs. 13(d) and 13(e)], each individual trajectory follows roughly the averaged trajectory and contains a very large number of different frequencies. This shows that the interaction "mixes" many of the nonperturbed levels, deleting the discrete structure of the spectrum.

Therefore, we can say that our master equation (31) or (32) holds with a given accuracy for a majority of individual trajectories if $\lambda$ is small enough satisfying $\lambda \geqslant C N^{-\nu}$ with $\nu<\frac{1}{2}$ and a constant $C>0$, in the limit $N \rightarrow \infty$.

\section{THE VERY LONG TIME BEHAVIOR}

We here focus on the very long time behavior of our model, in other words on its equilibrium properties.

\section{A. Equilibrium values of the system observables}

Let us consider the spin observable $\hat{\sigma}_{z}$ of the two-level system. This observable evolves in time according to

$$
\begin{aligned}
\left\langle\hat{\sigma}_{z}\right\rangle(t) & =\operatorname{Tr} \hat{\rho}(0) e^{i \hat{H}_{\mathrm{tot}} t} \hat{\sigma}_{z} e^{-i \hat{H}_{\mathrm{tot}} t} \\
& =\sum_{\alpha, \alpha^{\prime}}\left\langle\alpha|\hat{\rho}(0)| \alpha^{\prime}\right\rangle\left\langle\alpha^{\prime}\left|\hat{\sigma}_{z}\right| \alpha\right\rangle e^{i\left(E_{\alpha}-E_{\alpha^{\prime}}\right) t} .
\end{aligned}
$$

The time-averaged value of $\left\langle\hat{\sigma}_{z}\right\rangle(t)$ is obtained performing the following time average:

$$
\left\langle\hat{\sigma}_{z}\right\rangle_{\infty}=\lim _{T \rightarrow \infty} \frac{1}{T} \int_{0}^{T} d t\left\langle\hat{\sigma}_{z}\right\rangle(t)=\sum_{\alpha}\langle\alpha|\hat{\rho}(0)| \alpha\rangle\left\langle\alpha\left|\hat{\sigma}_{z}\right| \alpha\right\rangle .
$$

We see that this time averaged value clearly depends on the initial condition. 

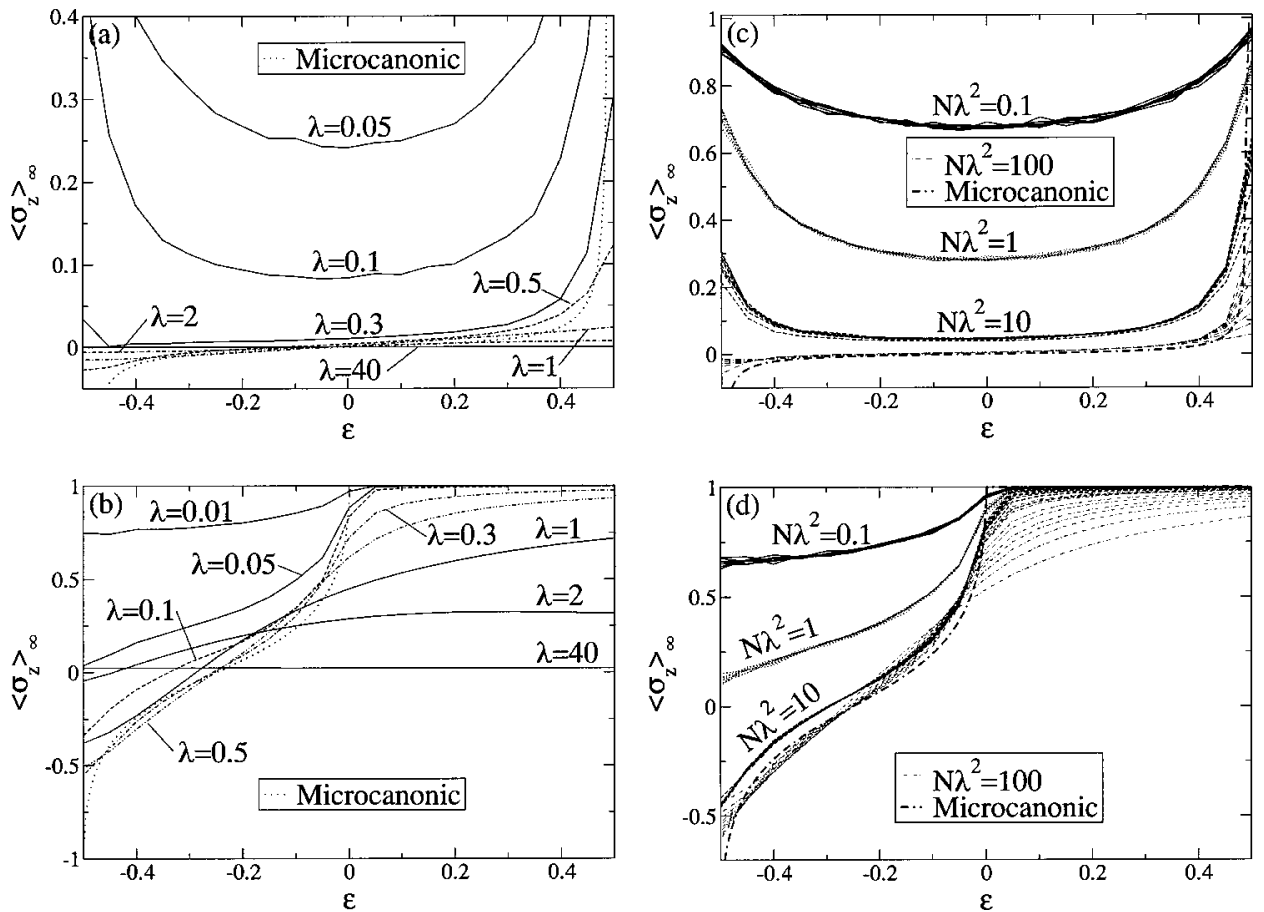

FIG. 14. Comparison between the equilibrium value of $\left\langle\hat{\sigma}_{z}\right\rangle_{\infty}$ given by the Pauli equation and the exact values given by the time averaged value for different values of $\lambda$. These equilibrium values are depicted as a function of the initial microcanonical energy $\epsilon$ of the environment. $\delta \epsilon=0.05$ in all figures. The parameter values are (a) $\Delta=0.01$ and $N=500 ;$ (b) $\Delta=0.5$ and $N=500$; (c) $\Delta=0.01$ and $N=200-2000 ;$ (d) $\Delta=0.5$ and $N=200-2000$.

An important result, that holds everywhere on the parameter space and for all kinds of initial conditions, is that the observed equilibrium value given by the exact von Neumann equation corresponds very well to the time-averaged value (55). Therefore, the study of the equilibrium properties of systems as ours is equivalent to studying the time averaged quantities (55).

Let us note that the standard initial condition we used till now is a microcanonical distribution around energy $\epsilon$ for the environment and an upper state for the system formally given by

$$
\hat{\rho}(0)=|1\rangle\left\langle 1\left|\otimes \frac{\delta\left(\hat{H}_{B}-\epsilon\right)}{n(\epsilon)}=\sum_{n} \frac{\delta\left(E_{n}-\epsilon\right)}{n(\epsilon)}\right| 1 n\right\rangle\langle 1 n| .
$$

Therefore, we have that

$$
\langle\alpha|\hat{\rho}(0)| \alpha\rangle=\sum_{n} \frac{\delta\left(E_{n}-\epsilon\right)}{n(\epsilon)}|\langle\alpha \mid 1 n\rangle|^{2} .
$$

An interesting point is to understand when the Markovian perturbative equation gives the correct equilibrium value. We want, therefore, to compare (55) with (37). This is done in Fig. 14 where we plotted the time averaged value $\left\langle\hat{\sigma}_{z}\right\rangle_{\infty}$ as a function of the initial energy of the environment $\epsilon$ [the initial value of the total system being (56)]. The different curves in Figs. 14(a) and 14(b) correspond to different values of $\lambda$. We compare these curves to the $\lambda$ independent curves given by the Markovian perturbative equation (37). As expected from our precedent study of the dynamics, we find again that, when $\lambda$ is too small, the "mixing" between the nonperturbed levels is not sufficient inside the microcanonical energy shell and the Markovian perturbative results overestimate the equilibrium values. If $\lambda$ is too large, the Markovian pertur- bative equation gives again bad results. The Markovian perturbative equilibrium values are correct in a characteristic region of $\lambda$. The beginning of this region corresponds, in the SOE, to the critical value of $\lambda$ at which the transition occurs from the localized to the Lorentzian regimes. The end of this region corresponds to $\lambda$ values that cannot be considered any more as perturbative. Figures 14(c) and 14(d) show that $\left\langle\hat{\sigma}_{z}\right\rangle_{\infty}$ scales like $\lambda^{2} N$. This again confirms our precedent analysis.

\section{B. Thermalization of the system}

One of the important questions is to understand the conditions under which the system thermalizes under the effect of a weak contact with the environment, or in other words, under which conditions the system relaxes to a canonical distribution corresponding to the microcanonical temperature of the environment.

We begin by recalling these conditions in the general case of a small system weakly interacting with its environment. The isolated total system is composed of the system and the environment and has the total energy

$$
E_{\mathrm{tot}}=\epsilon+e \text {. }
$$

$\epsilon$ is the energy of the environment and $e$ the energy of the system. We suppose that the contribution of the interaction energy between the environment and the system is negligible compared to the total energy. The microcanonical environment entropy is defined as

$$
S_{B}(\epsilon)=k \ln \Omega_{B}(\epsilon),
$$

where $\Omega_{B}(\epsilon)$ is the number of states of the environment available at energy $\epsilon$. This number can be related to the density of states of the environment $n_{B}(\boldsymbol{\epsilon})$ using the fact that 
$\Omega_{B}(\epsilon)=n_{B}(\epsilon) \delta \epsilon$, where $\delta \epsilon$ is a small energy interval but contains many states of the environment. The microcanonical temperature of the environment is given by

$$
\frac{1}{T_{B}(\epsilon)}=\frac{d S_{B}(\epsilon)}{d \epsilon} .
$$

It can be expanded in the system energy as

$$
T_{B}\left(\epsilon=E_{\mathrm{tot}}-e\right)=T_{B}\left(\epsilon=E_{\mathrm{tot}}\right)-e \frac{d T_{B}\left(\epsilon=E_{\mathrm{tot}}\right)}{d \epsilon}+\cdots,
$$

because we suppose that the system energy is much smaller than the environment energy. The specific heat capacity of the environment is

$$
\frac{1}{C_{v B}(\epsilon)}=\frac{d T_{B}(\epsilon)}{d \epsilon} .
$$

If the condition

$$
\left|C_{v B}(\epsilon)\right| \gg\left|\frac{e}{T_{B}(\epsilon)}\right|
$$

is satisfied, the temperature expansion can be truncated as follows:

$$
T_{B}\left(\epsilon=E_{\mathrm{tot}}-e\right)=T_{B}\left(\epsilon=E_{\mathrm{tot}}\right) .
$$

Therefore, we understand that if Eq. (63) is satisfied, the environment plays the role of a heat bath because its temperature is almost not affected by the system energy.

We suppose further that the interaction between the system and the environment, even if small, is able to make the total probability distribution microcanonical on the total energy shell at energy $E_{\text {tot }}$. We suppose also that the energy levels are discrete and, therefore, that $E_{\text {tot }}=E_{s}+E_{b}$ where $s$ and $b$ are discrete index's, respectively, for the system and the environment. Therefore, the probability $P_{S}\left(E_{s}\right)$ for the system being at energy $E_{s}$ is given by

$$
P_{S}\left(E_{s}\right)=\frac{\Omega_{B}\left(E_{b}=E_{\text {tot }}-E_{s}\right)}{\Omega_{\text {tot }}\left(E_{\text {tot }}\right)}
$$

where $\Omega_{B}\left(E_{b}\right)$ is the number of states of the environment available at energy $E_{b}$, and $\Omega_{\text {tot }}\left(E_{\text {tot }}\right)$ the number of states of the total system available at energy $E_{\text {tot }}$. Using Eqs. (60) and (63), one gets that

$$
\begin{aligned}
P_{S}\left(E_{s}\right) & =\frac{e^{(1 / k) S_{B}\left(E_{b}=E_{\mathrm{tot}}-E_{s}\right)}}{\Omega_{\mathrm{tot}}\left(E_{\mathrm{tot}}\right)} \\
& \simeq \frac{e^{(1 / k) S_{B}\left(E_{b}=E_{\mathrm{tot}}\right)-\left[E_{s} / k T_{B}\left(E_{B}=E_{\mathrm{tot}}\right)\right]}}{\Omega_{\mathrm{tot}}\left(E_{\mathrm{tot}}\right)} .
\end{aligned}
$$

Using the normalization $P_{S}\left(E_{s}\right)=1$, one finally gets the well-known canonical probability distribution for the system

$$
P_{S}\left(E_{s}\right)=\frac{e^{-\left[E_{s} / k T_{B}\left(E_{B}=E_{\mathrm{tot}}\right)\right]}}{Z},
$$

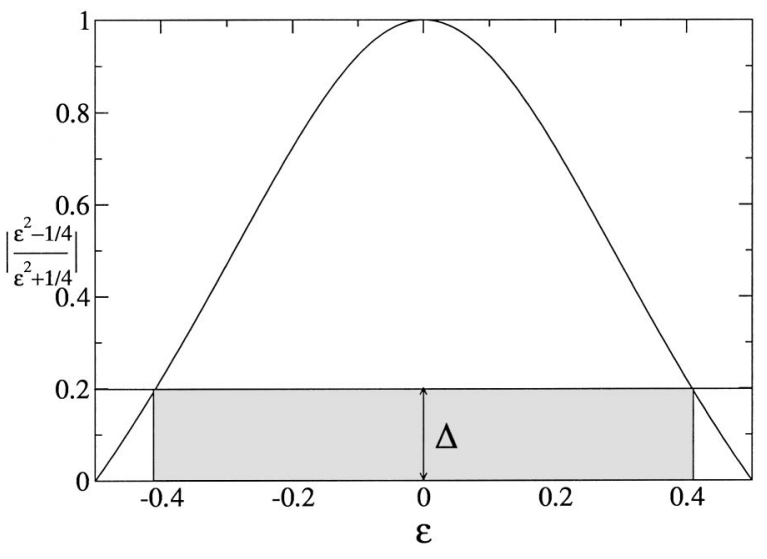

FIG. 15. Representation of the region where the thermalization condition $\left|\left(\epsilon^{2}-\frac{1}{4}\right) /\left(\epsilon^{2}+\frac{1}{4}\right)\right|>\Delta$ holds.

where $Z=\sum_{s} e^{-\left[E_{s} / k T_{B}\left(E_{B}=E_{\text {tot }}\right)\right]}$.

We conclude that two conditions are necessary in order to thermalize the system to a canonical probability distribution due to the contact with the environment: a large heat capacity of the environment $\left|C_{v B}(\epsilon)\right| \gg\left|e / T_{B}(\epsilon)\right|$ and a microcanonical distribution on the total energy shell.

Let us apply this result to the spin-GORM model. The density of states of the environment is

$$
n_{B}(\epsilon)=\frac{4 N}{\pi} \sqrt{\frac{1}{4}-\epsilon^{2}}
$$

Therefore the microcanonical temperature of the environment is

$$
T_{B}(\epsilon)=\frac{\epsilon^{2}-\frac{1}{4}}{k \epsilon}
$$

and the heat capacity is

$$
C_{v B}(\epsilon)=\frac{k \epsilon}{\epsilon^{2}+\frac{1}{4}}
$$

The canonical distribution of the populations of the system, at the microcanonical temperature of the environment, is given by

$$
\left\langle\hat{\sigma}_{z}\right\rangle_{T_{B}(\epsilon)}^{\operatorname{can}}=-\tanh \frac{\Delta}{2 k T_{B}(\epsilon)}=-\tanh \frac{\Delta \epsilon}{2\left(\epsilon^{2}-\frac{1}{4}\right)} .
$$

The first condition (63) in order to thermalize the system to a canonical probability distribution becomes

$$
\left|C_{v B}(\epsilon) T_{B}(\epsilon)\right|=\left|\frac{\epsilon^{2}-\frac{1}{4}}{\epsilon^{2}+\frac{1}{4}}\right| \gg \Delta
$$

and is depicted in Fig. 15. The second condition to have a microcanonical distribution on the total energy shell is satisfied (as we discussed in Sec. V A) when $\lambda^{2} N>1$. In this 

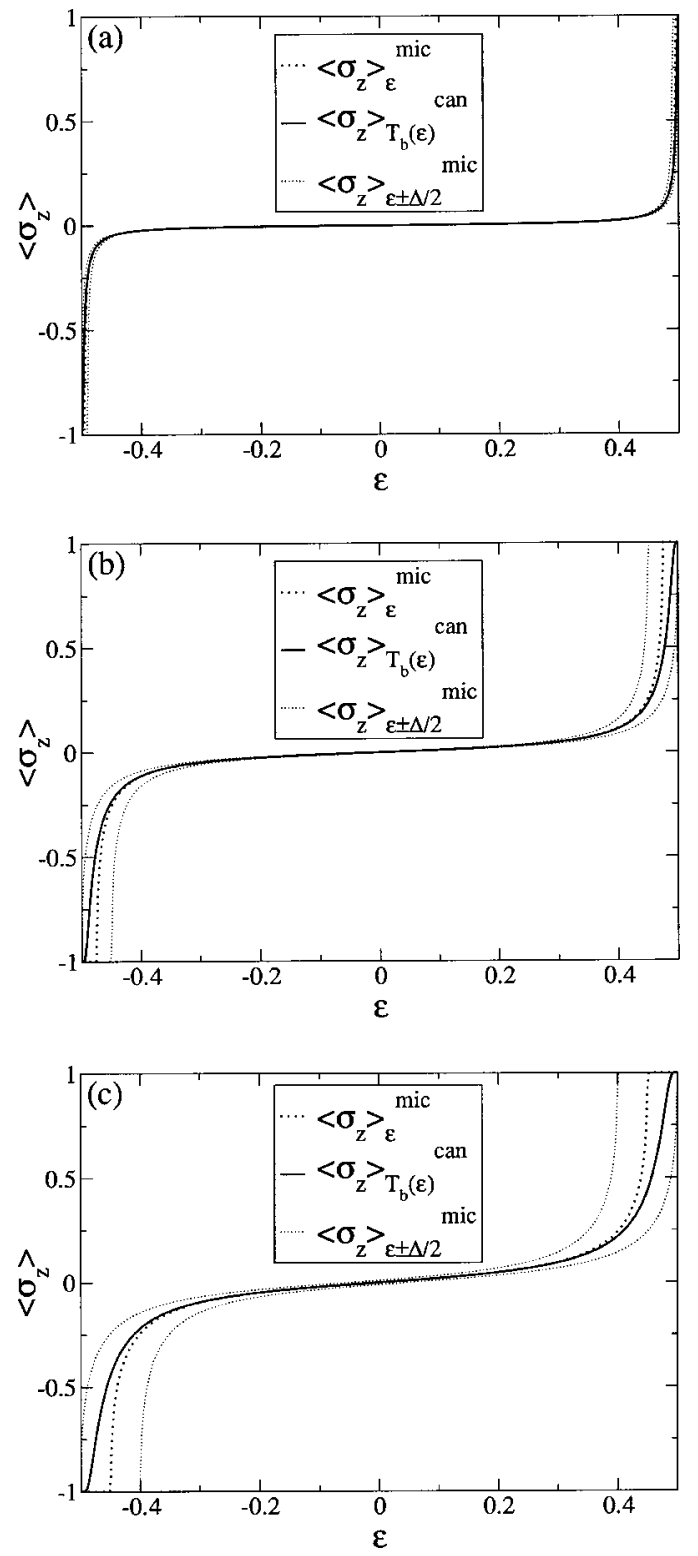

FIG. 16. Comparison between Eqs. (71) and (73) for different values of $\Delta$. The narrow dotted lines are plotted to show the undetermination around the environment energy. The parameter values are (a) $\Delta=0.01$; (b) $\Delta=0.05$; (c) $\Delta=0.1$. "mic" means microcanonic and "can" means canonic.

case, the populations of the system obey the microcanonical equilibrium value of the Markovian version of our perturbative equation (37), i.e.,

$$
\left\langle\hat{\sigma}_{z}\right\rangle_{\epsilon}^{\text {micro }}=\frac{\sqrt{\frac{1}{4}-\left(\epsilon-\frac{\Delta}{2}\right)^{2}}-\sqrt{\frac{1}{4}-\left(\epsilon+\frac{\Delta}{2}\right)^{2}}}{\sqrt{\frac{1}{4}-\left(\epsilon-\frac{\Delta}{2}\right)^{2}}+\sqrt{\frac{1}{4}-\left(\epsilon+\frac{\Delta}{2}\right)^{2}}} .
$$

If the two conditions are satisfied, then Eqs. (71) and (73) should be equal. The comparison between Eqs. (71) and (73) can be seen in Fig. 16 for different system energies. We see that the smaller the system energy is the better the comparison is. Therefore, we can conclude that under these two conditions $\left[\left|\left(\epsilon^{2}-\frac{1}{4}\right) /\left(\epsilon^{2}+\frac{1}{4}\right)\right| \gg \Delta\right.$ and $\left.\lambda^{2} N>1\right]$, the random matrices of the spin-GORM model can model an environment that behaves as a heat bath.

\section{Thermalization of the total system}

Until now, we have chosen initial conditions where the system is in the upper state with a microcanonical environment at a given energy, like in Eqs. (56) or (57). We now want to consider initial conditions where the system is again in the upper state but where the environment is at a given canonical temperature:

$$
\hat{\rho}(0)=|1\rangle\left\langle 1\left|\otimes \frac{e^{-\beta_{B} \hat{H}_{B}}}{Z_{B}}=\sum_{n} \frac{e^{-\beta_{B} E_{n}}}{Z_{B}}\right| 1 n\right\rangle\langle 1 n| .
$$

Therefore

$$
\langle\alpha|\hat{\rho}(0)| \alpha\rangle=\sum_{n} \frac{e^{-\beta_{B} E_{n}}}{Z_{B}}|\langle\alpha \mid 1 n\rangle|^{2} .
$$

It is important to notice that there is no statistical equivalence between the canonical and the microcanonical ensembles in the spin-GORM model (see Appendix B). Therefore, it is interesting to ask how the probability distribution looks like at equilibrium after the interaction.

One can clarify this point by plotting $\langle\alpha|\hat{\rho}(0)| \alpha\rangle$ versus energy. One uses the following energy representation:

$$
P(\varepsilon)=\sum_{\alpha} \delta\left(E_{\alpha}-\varepsilon\right)\langle\alpha|\hat{\rho}(0)| \alpha\rangle .
$$

If the total system thermalizes and reaches a canonical distribution for the total system at an effective temperature $\beta_{\mathrm{eff}}^{-1}$, one would have that

$$
P(\varepsilon)=\frac{e^{-\beta_{\mathrm{eff}} \varepsilon}}{Z_{\mathrm{tot}}},
$$

because

$$
\langle\alpha|\hat{\rho}(0)| \alpha\rangle=\frac{e^{-\beta_{\mathrm{eff}} E_{\alpha}}}{Z_{\mathrm{tot}}}
$$

As we shall see, it is the case if, again, $\lambda$ is large enough to induce "mixing" between the states $\lambda^{2} N>1$.

Indeed, one sees in Fig. 17(a) that, for $\lambda=0$, the states of the total system corresponding to the upper level of the system are exponentially populated and the ones corresponding to the lower level are not. When the interaction is turned on and increased, one can notice that the probability distribution starts to accumulate around a mean effective canonical distribution. As expected, this accumulation becomes significant when $\lambda^{2} N>1$ and, in this case, the total system can be considered as having thermalized. One can calculate the final effective temperature that the total system has reached after interaction. This effective temperature is depicted in Fig. 17(b) as a function of $\lambda$. The correlation coefficient indicates 



FIG. 17. In all the figures $\Delta=0.01, N=500$, and $\beta=2$. (a) Probability $P(\varepsilon)=\operatorname{Dist}(\varepsilon)$ of being in an eigenstate of the total system at equilibrium starting from the initial condition $\hat{\rho}(0)=|1\rangle\langle 1|$ $\otimes e^{-\beta \hat{H}_{B} / Z_{B}}$ with $1 / \beta=0.5$. (b) Effective temperature of the equilibrium probability distribution obtained by fitting a canonical distribution to the data of (a). (c) Comparison between the exact equilibrium population value and the canonical one computed with the effective temperature of plot (b). whether the exponential fit of the final effective temperature is good or not. The effective temperature obeys the following law: $\beta_{\text {eff }}=\beta_{i} /\left(1+\lambda^{2}\right)$. We also show in Fig. $17(\mathrm{c})$ the comparison between the time-averaged value of $\left\langle\hat{\sigma}_{z}\right\rangle$ and his canonical average computed with the effective temperature. One sees that, when $\lambda^{2} N>1$, both coincide.

This thermalization is not statistical in the sense of the equivalence between the ensembles. It is an intrinsic thermalization due to the complexity of the interaction between the states. This thermalization appears at a critical value of the coupling parameter when the interaction term becomes of the order of or larger than the mean level spacing of the total system.

\section{CONCLUSIONS}

In this paper, we have studied a system made of two parts: a two-level system interacting in a nondiagonal way with a complex environment modeled by Gaussian orthogonal random matrices. We began our study by analyzing the spectral properties of this model. We investigated the spectrum on a large energy scale with the averaged smooth density of states and on a finer energy scale with the eigenvalue diagrams, the shape of the eigenstates (SOE), the spacing distribution, and the asymptotic transition probability kernel (ATPK). We found a global repulsion as well as avoided crossings between the eigenvalues when the coupling parameter $\lambda$ was increased. We also showed the existence of three regimes (easy to distinguish in the SOE) that are important to describe the different qualitative behaviors of the model: the localized regime when the interaction between the levels is weaker than the mean level spacing $\lambda^{2} N \lesssim 1$ (giving rise to very narrow SOE), the Lorentzian regime when the interaction between the levels becomes larger than the mean level spacing $\lambda^{2} N>1$ (giving rise to a Lorentzian SOE), and the delocalized regime for very large $\lambda$ (giving rise to SOE spread over the whole spectrum).

After the spectral study, we started the study of the dynamics of the system populations. We defined different domains in the parameter space (see Fig. 1) and related them to the different relaxation behaviors of the system population induced by the interaction with the environment. For each of these domains we tested the validity of approximated population evolution equations. In the strong coupling limit, we identified a population relaxation regime described by a Bessel function: $\sim 2\left[J_{1}(\lambda t) / \lambda t\right](53)$ that scales in time according to $\lambda t$ and reaches an equilibrium distribution corresponding to the same probability of being in the upper and lower states of the system. In the small coupling limit and for small system energy, we obtained a Pauli-type equation (36)-(38) describing an exponential relaxation of the system population that scales in time according to $\lambda^{2} t$ and that reaches an equilibrium distribution depending on the system energy. The equilibrium value corresponds to a microcanonical probability distribution of being in a nonperturbed state of the total system inside the total energy shell. Finally, we showed the necessity of taking into account the nonMarkovian effects in the dynamics (which are important when the system energy becomes non-negligible in front of 
the environment energy) using a non-Markovian perturbative equation [(33) and (34)] derived by the authors in Ref. [17]. This equation is perturbative and therefore only valid for small coupling parameters. This equation describes the highly non-Markovian dynamics of the population (made of small and fast system oscillations damped on a time scale corresponding to the environment time scale) when the system energy becomes large in front of the environment energy. This equation also reduces to the Pauli-type equation in the opposite situation, when the system energy is small compared to the environment energy. The validity of these approximated equations depend on the parameter domain considered and are summarized in Fig. 12. An important result concerning the small coupling limit is that there exist lower and upper bounds on the coupling parameter values for which the perturbative equation holds. The lower bound depends on the spacing between the states of the total systems and therefore on the size $N$ of the random matrices modeling the environment. We showed that this lower bound is related to the transition between the localized and the Lorentzian regimes in the SOE and that this bound disappears when $N$ $\rightarrow \infty$.

Another important result concerns the equilibrium values of the spin-GORM model. We showed that they are very well reproduced by the time averaged quantities. Moreover, we showed that the spin-GORM model can, under two conditions, describe the thermalization of the system to a canonical energy probability distribution corresponding to the environment microcanonical temperature. The two conditions for this thermalization are a microcanonical probability distribution on the energy shell of the total system (that occurs when $\left.\lambda^{2} N>1\right)$ and a large heat capacity of the environment compared to the ratio between the characteristic system energy and the environment temperature.

Finally, we showed that the spin-GORM model can undergo an intrinsic thermalization due to the complex interaction between the states, and reach an overall thermal canonical distribution. This thermalization again occurs when the coupling parameter is large enough (i.e., larger than the mean level spacing of the total system) to "mix" the levels.

\section{ACKNOWLEDGMENTS}

The authors thank Professor G. Nicolis for support and encouragement in this research, as well as D. Cohen for several very fruitful discussions during his visit to Brussels. M.E. also wants to thank I. de Vega for her interesting comments on the spin-GORM model. M.E. is supported by the Fond pour la Formation à la Recherche dans l'Industrie et dans l'Agriculture, and P.G. by the National Fund for Scientific Research (FNRS Belgium).

\section{APPENDIX A: GAUSSIAN ORTHOGONAL RANDOM MATRICES (GORM)}

A Gaussian orthogonal random matrix (GORM) $\hat{Y}$ is characterized by $M$, the size of the matrix, and by the parameter $a_{\hat{Y}}$, which enters the Gaussian probability distribution $P(\hat{Y})=C e^{-\left(a_{\hat{Y}} / 2\right) \operatorname{Tr}\left(\hat{Y}^{2}\right)}$ of the whole matrix. The statistical properties of a GORM are preserved under orthogonal transformations. Because the matrix is orthogonal, each nondiagonal element $Y_{i j}$ is equal to its transposed $Y_{j i}$. The $M(M+1) / 2$ independent matrix elements of $\hat{Y}$ are Gaussian random numbers of mean zero. The standard deviation of the nondiagonal matrix elements $\sigma_{N D}^{\hat{Y}}$ and the standard deviation of the diagonal matrix element $\sigma_{D}^{\hat{Y}}$ are related to $a_{\hat{Y}}$ by

$$
\sigma_{D}^{\hat{Y}}=\sqrt{2} \sigma_{N D}^{\hat{Y}}=\sqrt{\frac{1}{a_{\hat{Y}}}} .
$$

The density of states of the GORM $\hat{Y}$ is defined by

$$
d(E)=\sum_{i=1}^{M} \delta\left(E-E_{i}\right)
$$

and the smoothed density of states by

$$
\bar{d}(E)=\lim _{\epsilon \rightarrow 0} \frac{1}{\epsilon} \int_{E-(\epsilon / 2)}^{E+(\epsilon / 2)} d(E) d E,
$$

where $\epsilon$ is a small energy interval which is large enough to contain many states in order for $\bar{d}(E)$ to be smooth. The averaged smoothed density of states is an ensemble average of $\chi$ realizations of the GORM. Such an ensemble is called the Gaussian orthogonal ensemble (GOE). It is well known [14-16] that the ensemble averaged smoothed density of states $\langle\bar{d}(E)\rangle_{\chi}$ obey the Wigner semicircular law in the limit $x \rightarrow \infty$ :

$$
\langle\bar{d}(E)\rangle_{\infty}= \begin{cases}\frac{a_{\hat{Y}}}{\pi} \sqrt{\frac{2 M}{a_{\hat{Y}}}-E^{2}} & \text { if }|E|<\sqrt{\frac{2 M}{a_{\hat{Y}}}}, \\ 0 & \text { if }|E| \geqslant \sqrt{\frac{2 M}{a_{\hat{Y}}}}\end{cases}
$$

The domain of energy where the eigenvalues are distributed (i.e., the width of the semicircular law) is $\mathcal{D} Y=\sqrt{8 M / a_{\hat{Y}}}$. Notice that when $M \rightarrow \infty, \bar{d}(E) \rightarrow\langle\bar{d}(E)\rangle_{\infty}$, and therefore $\bar{d}(E)$ follows the semicircular law. The following notation is used in the present paper: $n^{w}(E)=\langle\bar{d}(E)\rangle_{\infty}$.

\section{APPENDIX B: EQUIVALENCE BETWEEN ENSEMBLES FOR THE ENVIRONMENT}

Let us consider a system interacting with its environment. The environment is in a canonical distribution at temperature $T_{\text {can }}=1 / k \beta$. The evolution of a system observable is given by

$$
\left\langle\hat{A}_{S}\right\rangle^{\beta}(t)=\operatorname{Tr} \hat{\rho}_{S}(0) \frac{e^{-\beta \hat{H}_{B}}}{Z_{B}} e^{i \hat{H}_{\mathrm{tot}} \hat{A}_{S}} e^{-i \hat{H}_{\mathrm{tot}}{ }^{t}}
$$

On the other hand, if the environment is in a microcanonical ensemble, the evolution of the system observable is given by 


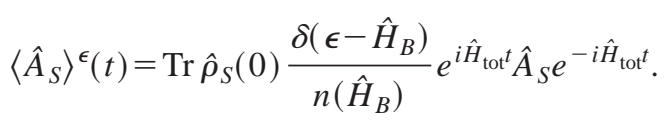

One therefore sees that

$$
\left\langle\hat{A}_{S}\right\rangle^{\beta}(t)=\int d \epsilon n(\epsilon) \frac{e^{-\beta \epsilon}}{Z_{B}}\left\langle\hat{A}_{S}\right\rangle^{\epsilon}(t) .
$$

The statistical equivalence between the canonical and microcanonical ensembles, $\left\langle\hat{A}_{S}\right\rangle^{\beta}(t)=\left\langle\hat{A}_{S}\right\rangle^{\epsilon^{\prime}}(t)$, thus occurs when

$$
n(\epsilon) \frac{e^{-\beta \epsilon}}{Z_{B}} \approx \delta\left(\epsilon-\epsilon^{\prime}\right) .
$$

One understands that this equivalence is qualitatively satisfied when $n(\epsilon)$ is an increasing function of $\epsilon$. In this case, $n(\epsilon)\left(e^{-\beta \epsilon} / Z_{B}\right)$ is a sharply peaked function. In order to find the maximum of $n(\epsilon)\left(e^{-\beta \epsilon} / Z_{B}\right)$, we require the vanishing of the derivative of its logarithm $(\partial / \partial \epsilon) \ln \left[n(\epsilon)\left(e^{-\beta \epsilon} / Z_{B}\right)\right]$ $=0$. We find that

$$
\frac{\partial}{\partial \epsilon} S\left(\epsilon_{\max }\right) \equiv \frac{1}{T_{\text {micro }}\left(\epsilon_{\max }\right)} \approx \frac{1}{T_{\text {can }}},
$$

where the microcanonical entropy is given by

$$
S(\epsilon)=k \ln n(\epsilon) \delta \epsilon .
$$

where $\delta \epsilon$ is a small energy shell containing many levels. This shows that if $n(\epsilon)\left(e^{-\beta \epsilon} / Z_{B}\right)$ is a sharply peaked function around $\epsilon_{\max }$, the canonical average at temperature $T_{\text {can }}$ is equivalent to the microcanonical average at the energy $\epsilon_{\max }$ corresponding to the microcanonical temperature $T_{\text {micro }}(\epsilon)=T_{\text {can }}$.

For the spin-GORM model, there is no equivalence between the canonical and the microcanonical ensembles. It is due to the fact that the semicircular energy distribution is not an increasing function of the energy. In this sense, this shows that the semicircular energy distribution does not describe a usual environment.

To complete our reasoning, we notice that the maximum of $n(\epsilon)\left(e^{-\beta \epsilon} / Z_{B}\right)$ is given by

$$
\epsilon_{\max }=\frac{1-\sqrt{1+\beta^{2}}}{2 \beta},
$$

so that $\epsilon_{\max }=0$ for $\beta \rightarrow 0$ and $\epsilon_{\max }=-\frac{1}{2}$ for $\beta \rightarrow \infty$.

\section{APPENDIX C: PERTURBATION THEORY}

There is no analytical way of getting a general form of the eigenvalues $E_{\alpha}$ of the total system, but the three terms in Eq. (5) have different orders of magnitude depending of the value of the parameters $\Delta$ and $\lambda$. The system and the environment Hamiltonians are, respectively, of order $\Delta$ and 1 while the coupling term is of order $\lambda$. Therefore, we can examine the different extreme cases that can be treated perturbatively.

\section{1. $\Delta, 1 \gg \lambda$}

When the system and the environment Hamiltonians are larger than the interaction term in Eq. (5), we can treat the interaction term in a perturbative way, taking the system and the environment Hamiltonian as reference,

$$
\hat{H}_{0}|s b\rangle=E_{s b}^{0}|s b\rangle,
$$

where we replaced the index $n$ by the two indices $s, b$. The perturbed energy is given to the second order by

$$
E_{\alpha}=E_{s, b}=\frac{\Delta}{2} s+E_{b}^{B}+\lambda^{2} \sum_{b^{\prime} \neq b} \frac{\left|\left\langle b^{\prime}|\hat{B}| b\right\rangle\right|^{2}}{E_{b}^{B}-E_{b^{\prime}}^{B}+s \Delta}+O\left(\lambda^{4}\right) .
$$

We notice that the first nonzero correction to the nonperturbed eigenstate is of order $\lambda^{2}$.

\section{2. $\lambda \gg 1, \Delta$}

When $\lambda$ is large compared to $\Delta$ and 1 in Eq. (5), it is possible to consider the interaction term as the reference Hamiltonian and to treat $\hat{H}_{S}$ and $\hat{H}_{B}$ as small perturbation. Transforming Eq. (5) by a unitary matrix acting only on the system degree of freedom, we get

$$
\hat{H}_{\text {tot }}=\frac{\Delta}{2} \hat{\sigma}_{x}+\hat{H}_{B}+\lambda \hat{\sigma}_{z} \hat{B} .
$$

The nonperturbed reference Hamiltonian is, therefore,

$$
\hat{\tilde{H}}_{0}=\lambda \hat{\sigma}_{z} \hat{B}
$$

Let $E_{\kappa \eta}$ and $|\kappa \eta\rangle=|\kappa\rangle \otimes|\eta\rangle$ be, respectively, the eigenvalues and eigenvectors of $\hat{H}_{0}$ :

$$
\hat{\tilde{H}}_{0}|\kappa \eta\rangle=\lambda \hat{B} \sigma_{z}|\kappa \eta\rangle=\lambda E_{\kappa \eta}|\kappa \eta\rangle=\lambda \kappa E_{\eta}|\kappa \eta\rangle,
$$

where $\eta=1, \ldots, N / 2$ and $\kappa= \pm 1$. The energy of the perturbed Hamiltonian is thus given to the second order perturbation in $1 / \lambda$ by

$$
\begin{aligned}
\frac{E_{\alpha}}{\lambda}= & \kappa E_{\eta}+\frac{1}{\lambda}\left\langle\eta\left|\hat{H}_{B}\right| \eta\right\rangle \\
& +\frac{1}{\lambda^{2}} \sum_{\substack{\kappa^{\prime}, \eta^{\prime} \\
\neq \kappa, \eta}} \frac{\left|\frac{\Delta}{2}+\left\langle\eta\left|\hat{H}_{B}\right| \eta\right\rangle\right|^{2}}{E_{\kappa \eta}^{0}-E_{\kappa^{\prime} \eta^{\prime}}^{0}}+O\left(\frac{1}{\lambda^{3}}\right) .
\end{aligned}
$$

\section{3. $1 \gg \Delta, \lambda$}

In this case, the bath Hamiltonian is large compared to the system Hamiltonian and the interaction term so that they both can be considered as perturbations. We get 


$$
\begin{aligned}
E_{\alpha}= & E_{s, b}=\frac{\Delta}{2} s+E_{b}^{B}+\lambda^{2} \sum_{b^{\prime} \neq b} \frac{\left|\left\langle b^{\prime}|\hat{B}| b\right\rangle\right|^{2}}{E_{b}^{B}-E_{b^{\prime}}^{B}} \\
& +O\left(\Delta^{2}\right)+O\left(\lambda^{2}\right) .
\end{aligned}
$$

\section{4. $\Delta \gg 1, \lambda$}

We now suppose that the system Hamiltonian, taken as reference, is large compared to the environment Hamiltonian and the interaction term, so that these last two terms can be considered as perturbations. We then get

$$
\begin{aligned}
E_{\alpha}= & E_{s, b}=\frac{\Delta}{2} s+E_{b}^{B}+s \frac{\lambda^{2}}{\Delta} \sum_{b^{\prime} \neq b}\left|\left\langle b^{\prime}|\hat{B}| b\right\rangle\right|^{2} \\
& +O(1)+O\left(\lambda^{2}\right) .
\end{aligned}
$$

Two more situations, $1, \lambda \gg \Delta$ and $\Delta, \lambda \gg 1$, could be considered but cannot be treated perturbatively because no reference basis exists in which $\hat{H}_{B}$ and $\hat{B}$ are simultaneously diagonal.
[1] W.M. Gelbart, S.A. Rice, and K.F. Freed, J. Chem. Phys. 57, 4699 (1972).

[2] J.L. Lebowitz and L. Pastur, e-print math-ph/0307004.

[3] P.A. Mello, P. Pereyra, and N. Kumar, J. Stat. Phys. 51, 77 (1988).

[4] P. Pereyra, J. Stat. Phys. 65, 773 (1991).

[5] E. Lutz and H.A. Weidenmüller, Physica A 267, 354 (1998).

[6] D. Cohen, F.M. Izrailev, and T. Kottos, Phys. Rev. Lett. 84, 2052 (2000).

[7] D. Cohen and T. Kottos, e-print cond-mat/0302319.

[8] A. Suárez, R. Silbey, and I. Oppenheim, J. Chem. Phys. 97, 5101 (1992).

[9] A.J. Leggett, S. Chakravarty, A.T. Dorsey, M.P.A. Fisher, A. Garg, and W. Zwerger, Rev. Mod. Phys. 59, 1 (1987).

[10] P. Gaspard and M. Nagaoka, J. Chem. Phys. 111, 5668 (1999).

[11] T. Guhr, A. Müller-Groeling, and H.A. Weidenmüller, Phys. Rep. 299, 189 (1998).

[12] O. Bohigas, M.J. Giannoni, and C. Schmit, Phys. Rev. Lett. 52, 1 (1984).
[13] G. Benenti, G. Casati, and D. Shepelyansky, Eur. Phys. J. D 17, 265 (2001).

[14] M.L. Mehta, Random Matrices and the Statistical Theory of Energy Levels (Academic Press, New York, 1967).

[15] C.E. Porter, Statistical Theories of Spectra Fluctuations (Academic Press, New York, 1965).

[16] T.A. Brody, J. Flores, J.B. French, P.A. Mello, A. Pandey, and S.S.M. Wong, Rev. Mod. Phys. 53, 385 (1981).

[17] M. Esposito and P. Gaspard, Phys. Rev. E 68, 066112 (2003).

[18] A.G. Redfield, IBM J. Res. Dev. 1, 19 (1957).

[19] U. Weiss, Quantum Dissipative Systems, 2nd ed. (World Scientific, Singapore, 2000).

[20] W. Pauli, Festschrift zum 60. Geburtstage A. Sommerfelds (Hirzel, Leipzig, 1928).

[21] R.W. Zwanzig, in Lectures in Theoretical Physics Vol. III, edited by W.E. Brittin, B.W. Downs, and J. Downs (Interscience, London, 1961), pp. 106-141.

[22] D. Zubarev, V. Morozov, and G. Röpke, Statistical Mechanics of Nonequilibrium Processes (Akademie Verlag, Berlin, 1996). 